\title{
INVARIANT THEORY FOR COINCIDENTAL COMPLEX REFLECTION GROUPS
}

\author{
VICTOR REINER, ANNE V. SHEPLER, AND ERIC SOMMERS
}

\begin{abstract}
V.F. Molchanov considered the Hilbert series for the space of invariant skew-symmetric tensors and dual tensors with polynomial coefficients under the action of a real reflection group, and he speculated that it had a certain product formula involving the exponents of the group. We show that Molchanov's speculation is false in general but holds for all coincidental complex reflection groups when appropriately modified using exponents and co-exponents. These are the irreducible well-generated (i.e., duality) reflection groups with exponents forming an arithmetic progression and include many real reflection groups and all non-real Shephard groups, e.g., the Shephard-Todd infinite family $G(d, 1, n)$. We highlight consequences for the $q$-Narayana and $q$-Kirkman polynomials, giving simple product formulas for both, and give a $q$-analogue of the identity transforming the $h$-vector to the $f$-vector for the coincidental finite type cluster/Cambrian complexes of Fomin-Zelevinsky and Reading. We include the determination of the Hilbert series for the non-coincidental irreducible complex reflection groups as well.
\end{abstract}

\section{INTRODUCTION}

Molchanov 24 hypothesized a formula for the dimensions of invariants of certain finite real reflection groups acting on skew-symmetric tensors and dual tensors with polynomial coefficients. His formula gives evidence that Solomon's invariant theory [35] for differential forms may have an extension to mixed derivation differential forms. We examine these forms not just for real reflection groups, but for complex reflection groups in general and compute their Hilbert series. We reformulate Molchanov's hypothesis in terms of exponents and coexponents of the group. Although his formula and this reformulation do not hold for all real reflection groups, they do hold for the important class of coincidental reflection groups.

The invariant theory of reflection groups acting on $V=\mathbb{C}^{n}$ displays a wondrous numerology controlled by two sequences of positive integers, the exponents $e_{1} \leq e_{2} \leq \cdots \leq e_{n}$ and coexponents $e_{1}^{*} \leq e_{2}^{*} \leq \cdots \leq e_{n}^{*}$. Solomon's Theorem [35] gives the dimensions of $W$-invariant polynomial differential forms on $V$ entirely in terms of the exponents of the reflection group $W$; his proof extends to describe likewise the invariant derivation forms in terms of the coexponents (see 25]). The degrees are the integers $d_{i}=e_{i}+1$. Those reflection groups satisfying $e_{i}+e_{n+1-i}^{*}=d_{n}$ are called duality groups. These are precisely the well-generated reflection groups, i.e., those generated by $n=\operatorname{dim} V$ reflections, and include all Coxeter groups.

An irreducible duality group $W$ is coincidental if its exponents $\left(e_{1}, e_{2}, \ldots, e_{n}\right)$ form an arithmetic sequence $\left(e_{1}, e_{1}+a, e_{1}+2 a, \ldots, e_{1}+(n-1) a\right)$ for some positive integer $a$ which we call its exponent gap. The coincidental reflection groups are the Coxeter groups of types $A_{n}, B_{n} / C_{n}, I_{2}(m), H_{3}$, the monomial groups $G(d, 1, n)$, all irreducible duality groups in rank 2 , and the groups $G_{25}, G_{26}$, and $G_{32}$ in the Shephard-Todd classification [31. They include all non-Coxeter Shephard groups, that is, the symmetry groups of regular complex polytopes.

We extend Solomon's description for the Hilbert series for invariant differential forms, $\left(S\left(V^{*}\right) \otimes \wedge V^{*}\right)^{W}$, to invariant mixed derivation differential forms, $\left(S\left(V^{*}\right) \otimes \wedge V^{*} \otimes \wedge V\right)^{W}$. We use the elementary symmetric functions $\sigma_{r}\left(x_{1}, \ldots, x_{n}\right):=\sum_{1 \leq i_{1}<\cdots<i_{r} \leq n} x_{i_{1}} x_{i_{2}} \cdots x_{i_{r}}$ with the convention that $\sigma_{0}\left(x_{1}, \ldots, x_{n}\right) \equiv 1$.

Theorem 1.1. For any coincidental complex reflection group $W$ acting on $V=\mathbb{C}^{n}$,

$$
\operatorname{Hilb}\left(\left(S\left(V^{*}\right) \otimes \wedge V^{*} \otimes \wedge V\right)^{W}, q, t, s\right)=\sum_{r=0}^{n} s^{r} \sigma_{r}\left(q^{e_{1}^{*}}, \ldots, q^{e_{n}^{*}}\right) \frac{\prod_{i=1}^{r}\left(1+q^{-e_{i}^{*}} t\right) \prod_{i=1}^{n-r}\left(1+q^{e_{i}} t\right)}{\prod_{i=1}^{n}\left(1-q^{d_{i}}\right)}
$$

where the coefficient of $q^{i} t^{k} s^{r}$ in the Hilbert series is the dimension of $\left(S^{i}\left(V^{*}\right) \otimes \wedge^{k} V^{*} \otimes \wedge^{r} V\right)^{W}$.

Date: September 11, 2019

1991 Mathematics Subject Classification. 13A50, 05Axx, 20F55.

Key words and phrases. Reflection groups, invariant theory, Weyl groups, Coxeter groups, f-vector, h-vector.

First author partially supported by NSF grant DMS-1601961; second author partially supported by Simons Foundation Grant \#429539. 
Here we use the standard grading on $S\left(V^{*}\right)=\oplus_{i} S^{i}\left(V^{*}\right)$ by polynomial degree. We may reformulate Theorem 1.1 compactly using the $q$-Pochhammer notation defined by

$$
(z ; q)_{k}:=(1-z)(1-z q) \cdots\left(1-z q^{k-1}\right)
$$

and the $q$-binomial coefficient defined by

$$
\left[\begin{array}{l}
n \\
r
\end{array}\right]_{q}:=\frac{(q ; q)_{n}}{(q ; q)_{r}(q ; q)_{n-r}}
$$

For a coincidental reflection group $W$, since the coexponents $\left(e_{1}^{*}, e_{2}^{*} \ldots, e_{n}^{*}\right)=(1,1+a, 1+2 a, \ldots, 1+(n-1) a)$, we can use the well-known identity [21, Chap. I §2, Ex. 3]

$$
\sigma_{r}\left(1, q, q^{2}, \ldots, q^{n-1}\right)=q^{\left(\begin{array}{c}
r \\
2
\end{array}\right)}\left[\begin{array}{l}
n \\
r
\end{array}\right]_{q}
$$

to rewrite the $r^{\text {th }}$ elementary symmetric function appearing in the theorem as

$$
\sigma_{r}\left(q^{e_{1}^{*}}, \ldots, q^{e_{n}^{*}}\right)=\sigma_{r}\left(q^{1}, q^{1+a}, q^{1+2 a}, \ldots, q^{1+(n-1) a}\right)=q^{r} \cdot \sigma_{r}\left(q^{a}, q^{2 a}, \ldots, q^{(n-1) a}\right)=q^{r+a\left(\begin{array}{c}
r \\
2
\end{array}\right)}\left[\begin{array}{l}
n \\
r
\end{array}\right]_{q^{a}} .
$$

We focus on the summand $\wedge^{r} V$ in $\wedge V=\oplus_{r=0}^{n} \wedge^{r} V$ and give the following equivalent version of Theorem 1.1.

Theorem 1.1. For a coincidental complex reflection group $W$ with smallest exponent $e_{1}$, exponent gap a,

$$
\text { Hilb }\left(\left(S\left(V^{*}\right) \otimes \wedge V^{*} \otimes \wedge^{r} V\right)^{W}, q, t\right)=q^{r+a\left(\begin{array}{c}
r \\
2
\end{array}\right)}\left[\begin{array}{l}
n \\
r
\end{array}\right]_{q^{a}} \frac{\left(-t q^{e_{1}} ; q^{a}\right)_{n-r}\left(-t q^{-1} ; q^{-a}\right)_{r}}{\left(q^{e_{1}+1} ; q^{a}\right)_{n}} \quad \text { for } r=0, \ldots, n .
$$

In fact, we compile the data on the Hilbert series of $\left(S\left(V^{*}\right) \otimes \wedge V^{*} \otimes \wedge V\right)^{W}$ for all irreducible complex reflection groups $W$, not just the coincidental groups - see Section 3 for Shephard and Todd's infinite family $G(d e, e, n)$ of monomial groups and Section 11 for the exceptional groups.

The $q$-analogues of $f$-vectors and $h$-vectors. Theorem 1.1 suggests $q$-analogues of the $f$-vector and the $h$-vector appearing in the algebraic combinatorics of certain simple polytopes and simplicial spheres, as we will explain in Section 10. These vectors record the number of faces of each dimension and the Hilbert-Poincaré polynomial of the associated Stanley-Reisner ring. For a coincidental reflection group $W$, we rename the right side of Theorem 1.1 as follows:

$$
f_{r}(W ; q, t):=q^{r+a\left(\begin{array}{c}
r \\
2
\end{array}\right)}\left[\begin{array}{l}
n \\
r
\end{array}\right]_{q^{a}} \frac{\left(-t q^{e_{1}} ; q^{a}\right)_{n-r}\left(-t q^{-1} ; q^{-a}\right)_{r}}{\left(q^{e_{1}+1} ; q^{a}\right)_{n}} .
$$

We wish to relate $f_{r}(W ; q, t)$ to a second product:

$$
h_{r}(W ; q, t):=\left(-t q^{-a r-1}\right)^{n-r}\left[\begin{array}{l}
n \\
r
\end{array}\right]_{q^{a}} \frac{\left(-t q^{-1} ; q^{-a}\right)_{r}}{\left(q^{e_{1}+1} ; q^{a}\right)_{r}} .
$$

We will see in Section 10 that the specializations

$$
f_{r}:=\left[f_{r}\left(W ; q, q^{h+1}\right)\right]_{q=1} \quad \text { for Coxeter number } \quad h:=e_{n}+1
$$

give the number of faces of each dimension in the finite type cluster fans of Fomin and Zelevinsky $[8$, when $W$ is a Weyl group, or the Cambrian fans of Reading [27] when $W$ is a real reflection group. For simplicial fans or polytopes, a standard re-encoding gives the $f$-vector entries $f_{r}$ in terms of the $h$-vector entries $h_{r}$ :

$$
\sum_{r=0}^{n} s^{r} f_{r}=\sum_{r=0}^{n}(1+s)^{r} \cdot h_{r}
$$

In Section 9, we use Theorem 1.1 to prove a $q$-analogue (and even a $(q, t)$-analogue) of this standard encoding:

Theorem 1.5. For any coincidental reflection group $W$ with exponent gap a,

$$
\sum_{r=0}^{n} s^{r} f_{r}(W ; q, t)=\sum_{r=0}^{n}\left(-s q ; q^{a}\right)_{r} \cdot h_{r}(W ; q, t)
$$


In Section 10, we explain why specializing $t$ in $f_{r}(W ; q, t)$ and $h_{r}(W ; q, t)$ to certain powers of $q$ give the q-Catalan numbers, q-Kirkman numbers, and q-Narayana numbers arising previously in [2, 29, 36] and how these specialize further to the aforementioned $f$-vector and $h$-vector entries.

Outline. After recalling the numerology of reflection groups in Section 2, we show Theorem 1.1 directly for the infinite family $G(d, 1, n)$ and the Weyl groups of type $A$ in Section 3 using results of Kirillov and Pak 19 and Koike 20. We also give the Hilbert series explicitly for the groups $G(d e, e, n)$ in Section 3 We conjecture an explicit basis for $\left(S\left(V^{*}\right) \otimes \wedge V^{*} \otimes \wedge V\right)^{W}$ in Section 4 constructed from invariant differential operators for coincidental reflection groups; invariance of the alleged basis elements in Conjecture 4.1 is checked in Section 5 In Section 6, we use the Gutkin-Opdam Lemma to predict the sum of degrees of these alleged basis elements. Section 7 then outlines the proof of Theorem 1.1 and compares it to Molchanov's original hypothesis. It also explains how we used Mathematica to verify Conjecture 4.1 for the real reflection group $H_{3}$ and the Shephard groups $G_{25}, G_{26}, G_{32}$. We verify Conjecture 4.1 for rank 2 groups in Section 8 In Section 9, we use Theorem 1.1 to define the above $q$-analogues of the $f$-vector and $h$-vector, and we prove Theorem 1.5 giving a $(q, t)$-analogue of the transformation (1.4) that converts between $f$ and $h$. We explain how specializations of these $q$-analogues give known product formulas for $q$-Catalan, $q$-Kirkman, and $q$-Narayana numbers in Section 10 and explain connections to graded parking spaces. Lastly, in Section 11 we compile the Hilbert series of $\left(S\left(V^{*}\right) \otimes \wedge V^{*} \wedge V\right)^{W}$ for all of the exceptional irreducible complex reflection groups.

\section{INVARIANT THEORY OF REFLECTION GROUPS}

We begin by recounting some appearances of the exponents and coexponents in the invariant theory of reflection groups. Recall that a reflection on $V=\mathbb{C}^{n}$ is a linear transformation whose fixed point space is a hyperplane and a reflection group $W$ is a subgroup of $\operatorname{GL}(V)$ generated by reflections. We assume all reflection groups are finite. Consequently, we may take an inner product on $V$ with respect to which $W$ acts by isometries and fix a basis of $V$ so that the matrices giving the action are unitary. We write $\operatorname{det}=\operatorname{det}_{V}$ throughout for the determinant of elements of $W$ acting on $V$. A reflection group is a (finite) Coxeter group or real reflection group if it is generated by reflections on $\mathbb{R}^{n}$, which then act on $\mathbb{C}^{n}$ by extension of scalars.

A large body of literature describes the invariant theory of reflection groups acting on $V=\mathbb{C}^{n}$ in terms of two sequences of positive integers, the exponents $e_{i}$ and coexponents $e_{i}^{*}$ of $W$,

$$
e_{1} \leq e_{2} \leq \cdots \leq e_{n} \text { and } e_{1}^{*} \leq e_{2}^{*} \leq \cdots \leq e_{n}^{*},
$$

defined as follows. The dual action of $W$ on $V^{*}$ induces an action on the symmetric algebra

$$
S\left(V^{*}\right) \cong \mathbb{C}\left[x_{1}, \ldots, x_{n}\right]
$$

where $x_{1}, \ldots, x_{n}$ is the $\mathbb{C}$-basis for $V^{*}$ dual to a $\mathbb{C}$-basis $y_{1}, \ldots, y_{n}$ of $V$; the group $W$ acts on $S\left(V^{*}\right)$ via invertible linear substitutions of the variables $x_{1}, \ldots, x_{n}$. A theorem of Shephard and Todd [31 and of Chevalley [6] asserts that the $W$-invariant polynomials form a polynomial subalgebra:

$$
S\left(V^{*}\right)^{W}=\mathbb{C}\left[f_{1}, \ldots, f_{n}\right]
$$

for some homogeneous $f_{i}$ in $S\left(V^{*}\right)$ called basic invariants. Their polynomial degrees $d_{1} \leq \cdots \leq d_{n}$ are independent of the choice of the $f_{i}$. The exponents of $W$ are then just the integers $e_{i}:=d_{i}-1$.

More generally, we may define $U$-exponents for any $W$-representation $U$ by regarding the $W$-fixed space $\left(S\left(V^{*}\right) \otimes U\right)^{W}$ as a module over $S\left(V^{*}\right)^{W}$ via multiplication into the left tensor factor. This module is free of rank $\operatorname{dim} U$ by Chevalley's Theorem [6] (see [5. Prop. 4.3.3, eqn. (4.6)]) or by a result of Hochster and Eagon [15], and the $U$-exponents $e_{1}(U) \leq \cdots \leq e_{\operatorname{dim} U}(U)$ are the degrees of a homogeneous basis. Here, $S\left(V^{*}\right) \otimes U$ inherits the grading on $S\left(V^{*}\right)$ by polynomial degree. Note that these $U$-exponents are the degrees in which the representation $U^{*}$ appears in the coinvariant algebra $S\left(V^{*}\right) / S\left(V^{*}\right)_{+}^{W}$.

As a special case, the $V$-exponents are the coexponents $e_{i}^{*}=e_{i}(V)$. In other words, $\left(S\left(V^{*}\right) \otimes V\right)^{W}$ is a free module over $S\left(V^{*}\right)^{W}$ and one may choose a basis $\left\{\theta_{1}, \ldots, \theta_{n}\right\}$, called a set of basic derivations, with

$$
\theta_{i}=\sum_{j=1}^{n} \theta_{i}^{j} \otimes y_{j} \quad \text { for homogeneous } \theta_{i}^{j} \text { in } S\left(V^{*}\right) \text { of degree } e_{i}^{*} \text {. }
$$

When $W$ is irreducible, there is a unique smallest coexponent $e_{1}^{*}=1$ corresponding to the Euler derivation, $\theta_{1}=\theta_{E}:=\sum_{i=1}^{n} x_{i} \otimes y_{i}$, which is always $W$-invariant (see [28], for example). 
Solomon [35] considered the space of differential forms and showed that the exterior algebra $\wedge V^{*}$ tensored with $S\left(V^{*}\right)$ has $W$-fixed space which is an exterior algebra over $S\left(V^{*}\right)^{W}$ on exterior generators $\left\{d f_{1}, \ldots, d f_{n}\right\}$ :

$$
\left(S\left(V^{*}\right) \otimes \wedge V^{*}\right)^{W}=\bigwedge_{S\left(V^{*}\right)^{W}}\left\{d f_{1}, \ldots, d f_{n}\right\} \quad \text { where } \quad d f:=\sum_{i=1}^{n} \frac{\partial f}{\partial x_{i}} \otimes x_{i} .
$$

From this one can deduce that the exponents are alternatively defined as the $V^{*}$-exponents via $e_{i}:=e_{i}\left(V^{*}\right)$. Orlik and Solomon [25, Thm. 3.1] generalized Solomon's Theorem, implying as a special case that the exterior algebra $\wedge V$ tensored with $S\left(V^{*}\right)$ has $W$-fixed space which is also an exterior algebra over base ring $S\left(V^{*}\right)^{W}$, this time with exterior generators given by the basic derivations $\left\{\theta_{1}, \ldots, \theta_{n}\right\}$ in $\left(S\left(V^{*}\right) \otimes V\right)^{W}$ :

$$
\left(S\left(V^{*}\right) \otimes \wedge V\right)^{W}=\bigwedge_{S\left(V^{*}\right)^{W}}\left\{\theta_{1}, \ldots, \theta_{n}\right\} .
$$

Hilbert Series. The above structural results imply combinatorial descriptions for various Hilbert series $\operatorname{Hilb}(M, q):=\sum_{d \geq 0} \operatorname{dim} M_{d} \cdot q^{d}$ for graded (or doubly or triply graded) vector spaces $M=\bigoplus_{d \geq 0} M_{d}$. In each case, one compares the Hilbert series implied by variants on Molien's Theorem (see [37] or [5, Lemma 3.2.8]) to the expression implied by the above results on the structure of the various rings and modules. Appreviating $S=S\left(V^{*}\right)$, we observe the following.

- The Shephard-Todd-Chevalley Theorem on $S^{W}$ implies that

$$
\frac{1}{|W|} \sum_{w \in W} \frac{1}{\operatorname{det}(1-q w)}=\operatorname{Hilb}\left(S^{W}, q\right)=\frac{1}{\prod_{i=1}^{n}\left(1-q^{d_{i}}\right)} .
$$

- The definition of coexponents in terms of $(S \otimes V)^{W}$ implies that

$$
\frac{1}{|W|} \sum_{w \in W} \frac{\chi_{V}\left(w^{-1}\right)}{\operatorname{det}(1-q w)}=\operatorname{Hilb}\left((S \otimes V)^{W}, q\right)=\left(\sum_{i=1}^{n} q^{e_{i}^{*}}\right) \frac{1}{\prod_{i=1}^{n}\left(1-q^{d_{i}}\right)} .
$$

- Solomon's Theorem describing $\left(S \otimes \wedge V^{*}\right)^{W}$ implies this generalization of (2.4):

$$
\frac{1}{|W|} \sum_{w \in W} \frac{\operatorname{det}(1+t w)}{\operatorname{det}(1-q w)}=\operatorname{Hilb}\left(\left(S \otimes \wedge V^{*}\right)^{W}, q, t\right)=\prod_{i=1}^{n} \frac{1+q^{e_{i}} t}{1-q^{d_{i}}},
$$

where the coefficient of $q^{i} t^{k}$ in the Hilbert series is the dimension of $\left(S^{i} \otimes \wedge^{k} V^{*}\right)^{W}$.

- The Orlik-Solomon Theorem describing $(S \otimes \wedge V)^{W}$ implies this different generalization of (2.4):

$$
\frac{1}{|W|} \sum_{w \in W} \frac{\operatorname{det}\left(1+s w^{-1}\right)}{\operatorname{det}(1-q w)}=\operatorname{Hilb}\left((S \otimes \wedge V)^{W}, q, t\right)=\prod_{i=1}^{n} \frac{1+q^{e^{*}} s}{1-q^{d_{i}}},
$$

where the coefficient of $q^{i} s^{r}$ in the Hilbert series is the dimension of $\left(S^{i} \otimes \wedge^{r} V^{*}\right)^{W}$.

Duality groups. More recently, the first two authors 28] proved a structural statement in the invariant theory of duality groups, that is, reflection groups satisfying the exponent-coexponent duality

$$
e_{i}+e_{n+1-i}^{*}=h:=\max \left\{d_{i}\right\} .
$$

For any reflection group $W$, the space $\left(S\left(V^{*}\right) \otimes \wedge V^{*} \otimes V\right)^{W}$ is a module over the $S\left(V^{*}\right)^{W}$-exterior algebra

$$
\left(S\left(V^{*}\right) \otimes \wedge V^{*}\right)^{W}=\bigwedge_{S\left(V^{*}\right)^{W}}\left\{d f_{1}, \ldots, d f_{n}\right\}
$$

via multiplication in the first two tensor factors. In general, it is not free as a module over this exterior algebra. But when $W$ is a duality group, $\left(S\left(V^{*}\right) \otimes \wedge V^{*} \otimes V\right)^{W}$ is free as a module over the subalgebra

$$
\bigwedge_{S\left(V^{*}\right)^{W}}\left\{d f_{1}, \ldots, d f_{n-1}\right\}
$$

which omits the last exterior generator $d f_{n}$. This similarly implies a combinatorial Hilbert series: 
Theorem 2.8 (28]). For $W$ a duality group,

$$
\begin{aligned}
\frac{1}{|W|} \sum_{w \in W} \chi_{V}\left(w^{-1}\right) \frac{\operatorname{det}(1+t w)}{\operatorname{det}(1-q w)} & =\operatorname{Hilb}\left(\left(S\left(V^{*}\right) \otimes \wedge V^{*} \otimes V\right)^{W}, q, t\right) \\
& =\left(\sum_{i=1}^{n} q^{e_{i}^{*}}\right) \frac{\left(1+q^{-1} t\right) \prod_{i=1}^{n-1}\left(1+q^{e_{i}} t\right)}{\prod_{i=1}^{n}\left(1-q^{d_{i}}\right)} .
\end{aligned}
$$

Note that setting $t=0$ in (2.9) gives (2.5) for duality groups $W$.

The $W$-invariants of $S\left(V^{*}\right) \otimes \wedge V^{*} \otimes \wedge^{n} V$. The following theorem holds for all complex reflection groups and agrees with extraction of the coefficient of $s^{n}$ in Theorem 1.1. It follows from results of the second author [32, 33, but we include a proof since we have not seen it stated explicitly in the literature.

Theorem 2.10. For any complex reflection group $W$ acting on $V$,

$$
\frac{1}{|W|} \sum_{w \in W} \operatorname{det}\left(w^{-1}\right) \frac{\operatorname{det}(1+t w)}{\operatorname{det}(1-q w)}=\operatorname{Hilb}\left(\left(S\left(V^{*}\right) \otimes \wedge V^{*} \otimes \wedge^{n} V\right)^{W}, q, t\right)=\prod_{i=1}^{n} \frac{q^{e_{i}^{*}}+t}{1-q^{d_{i}}} .
$$

Proof. Since $\wedge^{n} V$ carries the determinant character det of $W$, the $S^{W}$-module $\left(S \otimes \wedge V^{*} \otimes \wedge^{n} V\right)^{W}$ for $S=S\left(V^{*}\right)$ is the space of $\operatorname{det}^{-1}$-relative invariants for $W$ acting on $S \otimes \wedge V^{*}$. It was shown in 32] (see also [34) that the det $^{-1}$-relative invariant 1-forms $\left(S \otimes V^{*}\right)^{\operatorname{det}^{-1}}$ form a free $S^{W}$-module and any basis $\left\{\omega_{1}, \ldots, \omega_{n}\right\}$ generates the $\operatorname{det}^{-1}$-relative invariant $p$-forms $\left(S \otimes \wedge^{p} V^{*}\right)^{\operatorname{det}^{-1}}$ freely over $S^{W}$ with basis

$$
\left\{\frac{1}{Q^{p-1}} \cdot \omega_{i_{1}} \wedge \cdots \wedge \omega_{i_{p}}: 1 \leq i_{1}<\cdots<i_{p} \leq n\right\} .
$$

Here $Q:=\prod_{H} \ell_{H}$ is the $\operatorname{det}^{-1}$-relative invariant in $S$ of lowest degree, namely, the product of the linear forms $\ell_{H}$ whose vanishings define the reflecting hyperplanes $H$ of $W$. Thus $Q$ has degree equal to the number $N^{*}$ of reflecting hyperplanes, with known formula (e.g., [5, §4.5.5, Remark 4.48])

$$
\operatorname{deg}(Q)=: N^{*}=e_{1}^{*}+\cdots+e_{n}^{*} .
$$

If $\omega_{1}, \ldots, \omega_{n}$ are homogeneous with polynomial degrees $m_{1}, \ldots, m_{n}$, (so each $\omega_{i}$ lies in $S^{m_{i}} \otimes V^{*}$ ), then

$$
\begin{aligned}
\frac{\operatorname{Hilb}\left(\left(S \otimes \wedge V^{*} \otimes \wedge^{n} V\right)^{W}, q, t\right)}{\operatorname{Hilb}\left(S^{W}, q\right)} & =q^{\operatorname{deg}(Q)} \sum_{p=0}^{n} t^{p} \sum_{1 \leq i_{1}<\cdots<i_{p} \leq n} q^{m_{i_{1}}+\cdots+m_{i_{p}}-p \operatorname{deg}(Q)} \\
& =q^{\operatorname{deg}(Q)} \prod_{i=1}^{n}\left(1+t q^{m_{i}-\operatorname{deg}(Q)}\right)=\prod_{i=1}^{n}\left(q^{e_{i}^{*}}+t q^{e_{i}^{*}+m_{i}-\operatorname{deg}(Q)}\right) .
\end{aligned}
$$

Since $\operatorname{Hilb}\left(S^{W}, q\right)=\prod_{i=1}^{n} \frac{1}{1-q^{d_{i}}}$, the theorem will follow if we show that one can index so that

$$
m_{i}=\operatorname{deg}(Q)-e_{i}^{*}=\left(e_{1}^{*}+\cdots+e_{n}^{*}\right)-e_{i}^{*}
$$

for $i=1,2, \ldots, n$. To see this, we proceed as in the proof of [33, Cor. 4]. First note that the nondegenerate pairing $V \otimes \wedge^{n-1} V \rightarrow \wedge^{n} V$ is $W$-equivariant, where $\wedge^{n} V$ carries the character det. This implies $\wedge^{n-1} V \cong$ $V^{*} \otimes$ det as $W$-representations. Therefore the $S^{W}$-module of $\operatorname{det}^{-1}$-relative invariants in $S \otimes V^{*}$, which is isomorphic to the $W$-invariants $\left(S \otimes V^{*} \otimes \operatorname{det}\right)^{W}$, is also isomorphic to the $W$-invariants $\left(S \otimes \wedge^{n-1} V\right)^{W}$. However, by (2.3), the latter has $S^{W}$-basis $\left\{\theta_{1} \wedge \cdots \wedge \hat{\theta}_{i} \wedge \cdots \wedge \theta_{n}: i=1,2, \ldots, n\right\}$, whose elements indeed have degrees $\left(e_{1}^{*}+\cdots+e_{n}^{*}\right)-e_{i}^{*}$.

Invariant derivation differential forms. Theorem 1.1 describes the (triply-graded) Hilbert series

$$
\text { Hilb }\left(\left(S\left(V^{*}\right) \otimes \wedge V^{*} \otimes \wedge V\right)^{W}, q, t, s\right)=\frac{1}{|W|} \sum_{r=0}^{n} \sum_{w \in W} \frac{\operatorname{det}(1+t w) \operatorname{det}\left(1+s w^{-1}\right)}{\operatorname{det}(1-q w)},
$$

where the coefficient of $q^{i} t^{k} s^{r}$ is the dimension of $\left(S^{i}\left(V^{*}\right) \otimes \wedge^{k} V^{*} \otimes \wedge^{r} V\right)^{W}$, in terms of exponents and coexponents and specializes to all of (2.4), (2.5), (2.6), (2.7), (2.9) and Theorem 2.10. However, it applies only to the subfamily of coincidental reflection groups, described further here. 
Coincidental groups. A reflection group $W$ is coincidental if it is an irreducible duality group whose exponents (or equivalently, its degrees, or coexponents, or codegrees) form an arithmetic sequence. The coincidental reflection groups comprise (using notation from the classification of Shephard and Todd [31])

- the real reflection groups $A_{n}, B_{n} / C_{n}, I_{2}(m), H_{3}$,

- the monomial groups $G(d, 1, n)$,

- all rank 2 duality groups, namely, $G_{4}, G_{5}, G_{6}, G_{8}, G_{9}, G_{10}, G_{14}, G_{16}, G_{17}, G_{18}, G_{20}$, and $G_{21}$, and

- the groups $G_{25}, G_{26}$, and $G_{32}$.

Note that coincidental groups include all Shephard groups that are not Coxeter groups. Among the CoxeterShephard groups, coincidental groups exclude type $D_{n}$ for $n \geq 4$ and the real exceptional groups, $E_{6}, E_{7}$, $E_{8}, F_{4}, H_{4}$, i.e., those groups whose Coxeter diagram contains one of $D_{4}, F_{4}$, or $H_{4}$ as a subdiagram. The coincidental groups have made multiple appearances recently, for example, in the work of Miller [22, Thm. 14], 23, Thm. 2]. See [13, §5] for examples of real coincidental types in the literature.

Numerology of coincidental groups is governed by two parameters, the smallest exponent $e_{1}$ and the gap

$$
a:=d_{i}-d_{i-1}=e_{i}-e_{i-1}=e_{i}^{*}-e_{i-1}^{*}
$$

between any two successive exponents, or fundamental degrees, or coexponents:

$$
\begin{aligned}
& \left(d_{1}, d_{2}, \ldots, d_{n}\right)=\left(e_{1}+1, \quad e_{1}+1+a, \quad e_{1}+1+2 a, \ldots, \quad e_{1}+1+a(n-1)\right) \\
& \left(e_{1}, e_{2}, \ldots, e_{n}\right)=\left(\begin{array}{cccc}
e_{1}, & e_{1}+a, \quad e_{1}+2 a \quad, \ldots, \quad e_{1}+a(n-1)
\end{array}\right) \\
& \left(e_{1}^{*}, e_{2}^{*}, \ldots, e_{n}^{*}\right)=\left(\begin{array}{cccc}
1, & 1+a, & 1+2 a \quad, \ldots, & 1+a(n-1)
\end{array}\right) \text {. }
\end{aligned}
$$

\section{Type $A$ And the monomial groups}

We begin compiling our verification of Theorem 1.1 with the Weyl groups of type $A$ and the infinite family of Shephard-Todd groups $G(d, 1, n)$.

Recall that for positive integers $d, n$, the complex reflection group $G(d, 1, n)$ is the set of all $n \times n$ matrices in $\mathrm{GL}(V)=\mathrm{GL}_{n}(\mathbb{C})$ that are monomial (exactly one nonzero entry in each row and column) with nonzero entries all $d^{\text {th }}$ roots-of-unity in $\mathbb{C}$. Any element $w$ in $G(d, 1, n)$ maps the basis vectors $y_{1}, \ldots, y_{n}$ of $V=\mathbb{C}^{n}$ to $\zeta^{m_{1}} y_{\pi(1)}, \ldots, \zeta^{m_{n}} y_{\pi(n)}$ for some permutation $\pi=\pi(w)$ in the symmetric group $\mathfrak{S}_{n}=G(1,1, n)$, where $\zeta$ is the complex root-of-unity $e^{\frac{2 \pi i}{d}}$. In fact, $G(d, 1, n)=\mathfrak{S}_{n} \ltimes(\mathbb{Z} / d \mathbb{Z})^{n}$ since the map $w \mapsto \pi(w)$ is a surjective group homomorphism, $G(d, 1, n) \longrightarrow \mathfrak{S}_{n}$, whose kernel is the subgroup $(\mathbb{Z} / d \mathbb{Z})^{n}$ of diagonal matrices within $G(d, 1, n)$. We need to draw a distinction between the symmetric group $\mathfrak{S}_{n}$ acting as the permutation matrices $G(1,1, n)$ on one hand and acting as the Weyl group $A_{n-1}$ on the other hand:

- The group $G(1,1, n)$ acts on $V=\mathbb{C}^{n}$ reducibly, with fixed space $V^{\mathfrak{S}_{n}}=\mathbb{C}\left(y_{1}+\cdots+y_{n}\right)$.

- The group $W\left(A_{n-1}\right)$ acts irreducibly on the quotient space $V / \mathbb{C}\left(y_{1}+\cdots+y_{n}\right) \cong \mathbb{C}^{n-1}$.

For any finite group $W$, we introduce a shorthand notation for the Hilbert series describing the isotypic component in $S\left(V^{*}\right) \otimes \wedge V^{*}$ corresponding to a $W$-representation $M$ with character $\chi$. Again, we use a Molien type theorem to write this Hilbert series as a sum over group elements and abbreviate $S=S\left(V^{*}\right)$. We are interested in the special case when $\chi$ is the character of the $W$-representation $\wedge^{r} V$ giving (2.11).

Definition 3.1. For any finite subgroup $W$ of GL $(V)$ and any character $\chi$ of a $W$-module $M$, define

$$
\begin{aligned}
P_{W}(\chi ; q, t) & :=\operatorname{Hilb}\left(\left(S \otimes \wedge V^{*} \otimes M^{*}\right)^{W}, q, t\right)=\frac{1}{|W|} \sum_{w \in W} \chi(w) \frac{\operatorname{det}(1+t w)}{\operatorname{det}(1-q w)} \quad \text { and } \\
\chi_{S \otimes \wedge V^{*}}(q, t)(w) & :=\sum_{j=0}^{\infty} \sum_{k=0}^{n} q^{j} t^{k} \chi_{S^{j} \otimes \wedge^{k} V^{*}}(w) \quad \text { for } w \text { in } W .
\end{aligned}
$$

The second expression, $\chi_{S \otimes \wedge V^{*}}(q, t)(w)$, is a class function on $W$ with values in the ring $\mathbb{Z}[t][[q]]$. Notice that in terms of the usual inner product $\langle\cdot, \cdot\rangle_{W}$ on $W$-class functions,

$$
P_{W}(\chi ; q, t)=\left\langle\chi, \chi_{S \otimes \wedge V^{*}}(q, t)\right\rangle_{W} .
$$

The next two subsections review formulas for $P_{W}(\chi ; q, t)$ for $\chi$ a $W$-irreducible character for $W=W\left(A_{n-1}\right)$ and $G(d, 1, n)$. Since $\wedge^{r} V$ and $\left(\wedge^{r} V\right)^{*}$ are irreducible $W$-representations (see [18, §24-3]), we examine

$$
P_{W}\left(\left(\chi_{\wedge^{r} V}\right)^{*} ; q, t\right)=\operatorname{Hilb}\left(\left(S \otimes \wedge V^{*} \otimes \wedge^{r} V\right)^{W}, q, t\right)
$$


to verify Theorem 1.1 for the coincidental types $W\left(A_{n-1}\right)$ and $G(d, 1, n)$ with $d \geq 2$.

The Type A formula of Kirillov-Pak, Molchanov, Thibon, Gyoja-Nishiyama-Shimura. The following "hook-content" formula for $P_{W}(\chi ; q, t)$ when $W=\mathfrak{S}_{n}=G(1,1, n)$ is the building block for the rest. This formula was proven first by Kirillov and Pak [19, eqn. (4)] bijectively and then more algebraically by Molchanov [24, eqn. (2)]. It was also deduced from symmetric function identities by Thibon [39, Thm. 4.3] and by Gyoja, Nishiyama, and Shimura [14, eqn. (3.2)]. To state the formula, recall that the irreducible characters of the symmetric group $\mathfrak{S}_{n}$ can be indexed as $\left\{\chi^{\lambda}\right\}$ where $\lambda=\left(\lambda_{1} \geq \lambda_{2} \geq \cdots \geq 0\right)$ runs through the partitions of $n$, that is, $|\lambda|:=\sum_{i} \lambda_{i}=n$. Define $n(\lambda):=\sum_{i>1}(i-1) \lambda_{i}$. Recall also the notion of the Ferrers diagram for $\lambda$, containing the cells $x=(i, j)$ in row $i$ and column $j$ for $1 \leq j \leq \lambda_{i}$. The cell $x=(i, j)$ is said to have content $c(x):=j-i$ and hooklength $h(x):=\lambda_{i}+\#\left\{k: \lambda_{k} \geq i\right\}-j$.

Theorem 3.3. [14, 19, 24] For $W=\mathfrak{S}_{n}=G(1,1, n)$ acting on $V=\mathbb{C}^{n}$ and any character $\chi^{\lambda}$ of $W$,

$$
P_{\mathfrak{S}_{n}}\left(\chi^{\lambda} ; q, t\right)=q^{n(\lambda)} \prod_{x \in \lambda} \frac{1+t q^{c(x)}}{1-q^{h(x)}} .
$$

The theorem gives the following corollary for the irreducible action $A_{n-1}$ of the symmetric group $\mathfrak{S}_{n}$.

Corollary 3.4. For the Weyl group $W\left(A_{n-1}\right)$ acting on $V=\mathbb{C}^{n-1}$ and any irreducible character $\chi^{\lambda}$,

$$
\begin{aligned}
P_{W\left(A_{n-1}\right)}\left(\chi^{\lambda} ; q, t\right) & =\frac{1-q}{1+t} \quad P_{\mathfrak{S}_{n}}\left(\chi^{\lambda} ; q, t\right) \\
& =\frac{1-q}{1+t} q^{n(\lambda)} \prod_{x \in \lambda} \frac{1+t q^{c(x)}}{1-q^{h(x)}} .
\end{aligned}
$$

In particular, for $\chi^{\lambda}$ the character $\chi_{\wedge^{r} V}$ of the $W$-representation $\wedge^{r} V$ for some fixed $r=0, \ldots, n$,

$$
P_{W\left(A_{n-1}\right)}\left(\chi_{\wedge^{r} V} ; q, t\right)=q^{r+\left(\begin{array}{c}
r+1 \\
2
\end{array}\right)}\left[\begin{array}{c}
n-1 \\
r
\end{array}\right]_{q} \frac{(-t q ; q)_{n-r-1}\left(-t q^{-1} ; q^{-1}\right)_{r}}{(q ; q)_{n-1}}
$$

and the assertion of Theorem 1.1 holds for type $A_{n-1}$.

Proof. Equation (3.5) follows from the fact that a permutation matrix $\pi$ in $G(1,1, n)$ acts on $\mathbb{C}^{n}$ with one extra eigenvalue +1 compared to its action $w$ on $\mathbb{C}^{n-1}$ as an element in $W\left(A_{n-1}\right)$, and hence

$$
\frac{\operatorname{det}(1+t \pi)}{\operatorname{det}(1-q \pi)}=\frac{1+t}{1-q} \cdot \frac{\operatorname{det}(1+t w)}{\operatorname{det}(1-q w)} \text {. }
$$

Theorem 3.3 then gives (3.6). For (3.7), note that $\left(\chi_{\wedge^{r} V}\right)^{*}=\chi_{\wedge^{r} V}=\chi^{\lambda}$ for $\lambda=\left(n-r, 1^{r}\right)$, which has

$$
\begin{aligned}
n(\lambda) & =\left(\begin{array}{c}
r+1 \\
2
\end{array}\right), \\
\text { cell contents } c(x) & =(0,1,2, \ldots, n-1-r,-1,-2, \ldots,-r), \\
\text { hooklengths } h(x) & =(1,2, \ldots, n, 1,2, \ldots, r) .
\end{aligned}
$$

We apply (3.6) in this special case and obtain

$$
\begin{aligned}
P_{W\left(A_{n-1}\right)}\left(\left(\chi_{\wedge^{r} V}\right)^{*} ; q, t\right)=P_{W\left(A_{n-1}\right)}\left(\chi_{\wedge r} ; q, t\right) & =\frac{1-q}{1+t} q^{n(\lambda)} \prod_{x \in \lambda} \frac{1+t q^{c(x)}}{1-q^{h(x)}} \\
& =\frac{1-q}{1+t} q^{\left(\begin{array}{c}
r+1 \\
2
\end{array}\right)} \frac{1+t}{1-q^{n}} \prod_{i=1}^{n-r-1} \frac{1+t q^{i}}{1-q^{i}} \prod_{i=1}^{r} \frac{1+t q^{-i}}{1-q^{i}} \\
& =q^{\left(\begin{array}{c}
r+1 \\
2
\end{array}\right)} \frac{1-q}{1-q^{n}} \frac{(-t q ; q)_{n-r-1}}{(q ; q)_{n-r-1}} \frac{\left(-t q^{-1} ; q^{-1}\right)_{r}}{(q ; q)_{r}} \\
& =q^{\left(\begin{array}{c}
r+1 \\
2
\end{array}\right)} \frac{1-q}{1-q^{n}}\left[\begin{array}{c}
n-1 \\
r
\end{array}\right]_{q} \frac{(-t q ; q)_{n-r-1}\left(-t q^{-1} ; q^{-1}\right)_{r}}{(q ; q)_{n-1}} \\
& =q^{r+\left(\begin{array}{c}
r \\
2
\end{array}\right)}\left[\begin{array}{c}
n-1 \\
r
\end{array}\right] \begin{array}{c}
(-t q ; q)_{n-r-1}\left(-t q^{-1} ; q^{-1}\right)_{r} \\
\left(q^{2} ; q\right)_{n-1}
\end{array}
\end{aligned}
$$


using (1.2) in the penultimate equality.

Finally, to verify Theorem 1.1 for $W\left(A_{n-1}\right)$, we apply (3.2) and check that the last expression in (3.8) agrees with the expression in Theorem 1.1] (see (2.11)). This holds because $W\left(A_{n-1}\right)$ has rank $n-1$ and exponents

$$
\left(e_{1}, \ldots, e_{n-1}\right)=(1,2, \ldots, n-1)
$$

so that its smallest exponent is $e_{1}=1$ and the gap between exponents is $a=1$. We also use the fact that $W\left(A_{n-1}\right)$ is a real reflection group, so that $V \cong V^{*}$ and $e_{i}^{*}=e_{i}$.

Koike's formula for $G(d, 1, n)$. We review here a formula of Koike 20] generalizing to the monomial groups $W=G(d, 1, n)$ for $d \geq 2$ the calculation of $P_{W}(\chi, t)$ completed for $G(2,1, n)=W\left(B_{n}\right)$ by Kirillov and Pak [19, eqn. (6)]; see also Gyoja, Nishiyama, and Shimura [14, eqn. (3.9)] for the case of $W\left(B_{n}\right)$.

Fix $d \geq 2$, and let us abbreviate $W_{n}:=G(d, 1, n)$ acting on $V=\mathbb{C}^{n}$. The irreducible characters of $W_{n}$ can be indexed by d-multipartitions of $n$

$$
\underline{\lambda}=\left(\lambda^{(0)}, \lambda^{(1)}, \ldots, \lambda^{(d-1)}\right)
$$

in which each $\lambda^{(i)}$ is a partition of $n_{i}$ and $\sum_{i=0}^{d-1} n_{i}=n$. Denoting $\underline{n}:=\left(n_{0}, n_{1}, \ldots, n_{d_{1}}\right)$, let $W_{\underline{n}}$ be the subgroup of $W_{n}$ isomorphic to $W_{n_{0}} \times W_{n_{1}} \times \cdots \times W_{n_{d-1}}$ consisting of the block diagonal matrices in $W_{n}$ with diagonal block sizes specified by $\underline{n}$.

Recall that any element $w$ in $G(d, 1, n)$ can be written uniquely as $w=\operatorname{diag}(w) \cdot \pi(w)$, the product of a diagonal matrix $\operatorname{diag}(w)$ and a permutation matrix $\pi(w)$ in $G(1,1, n)$. This gives rise to a 1-dimensional character recording the determinant of the diagonal part of $w$ (i.e., the product of the nonzero entries in $w$ ):

$$
\epsilon: W_{n} \longrightarrow \mathbb{C}^{\times}, \quad w \mapsto \operatorname{det}(\operatorname{diag}(w)) .
$$

Given any $\mathfrak{S}_{n}$-character $\chi$, one can inflate it along $\pi$ to a $W_{n}$-character that we will denote $\chi \Uparrow_{\mathfrak{S}_{n}}^{W_{n}}$. Given any $W_{\underline{n}}$-character $\chi$, one can induce it up to $W_{n}$, giving a character that we will denote $\chi \uparrow_{W_{\underline{n}}}^{W_{n}}$. One then has the following description for the irreducible $W_{n}$-character indexed by $\underline{\lambda}$ as in (3.9):

$$
\chi^{\underline{\lambda}}=\left(\bigotimes_{i=0}^{d-1} \epsilon^{i} \otimes\left(\chi^{\lambda_{i}} \Uparrow_{\mathfrak{S}_{n_{i}}}^{W_{n_{i}}}\right)\right) \uparrow_{W_{\underline{n}}}^{W_{n}}
$$

Here $\epsilon$ is the same degree 1 character of $W_{n}$ restricted to each $W_{n_{i}}$, and $\epsilon^{i}$ is its $i^{\text {th }}$ tensor power.

We can now state Koike's result. We include a proof which is shorter and less computational than the one in [20, pp. 545-548], following the methodology of Kirillov and Pak.

Theorem 3.11. [20, Theorem 1] For any d-multipartition $\underline{\lambda}$ of $n$ as above,

$$
P_{W_{n}}\left(\chi^{\underline{\lambda}} ; q, t\right)=P_{\mathfrak{S}_{n_{0}}}\left(\chi^{\lambda^{(0)}} ; q^{d}, t q^{d-1}\right) \cdot \prod_{i=1}^{d-1} q^{n_{i}(d-i)} \cdot P_{\mathfrak{S}_{n_{i}}}\left(\chi^{\lambda^{(i)}} ; q^{d}, t q^{-1}\right) .
$$

Proof. (cf. [19, Proof of Lemma 1]) By Frobenius reciprocity,

$$
P_{W_{n}}\left(\chi^{\underline{\lambda}} ; q, t\right)=\left\langle\chi^{\underline{\lambda}}, \chi_{S \otimes \wedge V^{*}}(q, t)\right\rangle_{W_{n}}=\prod_{i=0}^{d-1}\left\langle\epsilon^{i} \otimes\left(\chi^{\lambda^{(i)}} \Uparrow_{\mathfrak{S}_{n_{i}}}^{W_{n_{i}}}\right), \chi_{S \otimes \wedge V^{*}}(q, t)\right\rangle_{W_{n_{i}}} .
$$

It remains to compute the $i^{\text {th }}$ factor in the product. For ease of notation, replace $n_{i}$ by $n$ and $\lambda^{(i)}$ by $\lambda$, so that we may rewrite the $i^{\text {th }}$ factor simply as

$$
\begin{aligned}
\left\langle\epsilon^{i} \otimes\left(\chi^{\lambda} \Uparrow \mathbb{S}_{n}^{W_{n}}\right), \chi_{S \otimes \wedge V^{*}}(q, t)\right\rangle_{W_{n}} & =\left\langle\chi^{\lambda} \Uparrow_{\mathfrak{S}_{n}}^{W_{n}}, \epsilon^{-i} \otimes \chi_{S\left(V^{*}\right) \otimes \wedge V^{*}}(q, t)\right\rangle_{W_{n}} \\
& =\left\langle\chi^{\lambda}, \chi_{\left(\epsilon^{-i} \otimes S\left(V^{*}\right) \otimes \wedge V^{*}\right)^{(\mathbb{Z} / d \mathbb{Z})^{n}}}(q, t)\right\rangle_{\mathfrak{S}_{n}} .
\end{aligned}
$$

Here the last equality uses a general adjointness statement (see, e.g., [11, §4.1.6]) for quotient groups $G \rightarrow G / N$, taking $(G, N, G / N)$ to be $\left(W_{n},(\mathbb{Z} / d \mathbb{Z})^{n}, \mathfrak{S}_{n}\right)$ : For any $G$-representation $A$, with residual $G / N$ representation on its $N$-fixed space $A^{N}$, and any $G / N$-representation $B$,

$$
\left\langle\chi_{B} \Uparrow_{G / N}^{G}, \chi_{A}\right\rangle_{G}=\left\langle\chi_{B}, \chi_{A^{N}}\right\rangle_{G / N}
$$


We next examine, for $0 \leq i \leq d-1$, the $(\mathbb{Z} / d \mathbb{Z})^{n}$-fixed space,

$$
\left(\epsilon^{-i} \otimes S\left(V^{*}\right) \otimes \wedge V^{*}\right)^{(\mathbb{Z} / d \mathbb{Z})^{n}}
$$

Note the following tensor product decomposition, compatible with the $(\mathbb{Z} / d \mathbb{Z})^{n}$-action:

$$
S\left(V^{*}\right) \otimes \wedge V^{*}=\bigotimes_{j=1}^{n} \mathbb{C}\left[x_{j}\right] \otimes \wedge \mathbb{C} x_{j}
$$

Since the character $\epsilon$ restricted to $(\mathbb{Z} / d \mathbb{Z})^{n}$ is just the tensor product of $\epsilon$ on each of the factors $\mathbb{Z} / d \mathbb{Z}$, the $(\mathbb{Z} / d \mathbb{Z})^{n}$-fixed space (3.14) is the tensor product of these $\mathbb{Z} / d \mathbb{Z}$-fixed spaces:

$$
\left(\epsilon^{-i} \otimes \mathbb{C}\left[x_{j}\right] \otimes \wedge \mathbb{C} x_{j}\right)^{\mathbb{Z} / d \mathbb{Z}}= \begin{cases}\left(\mathbb{C}\left[x_{j}^{d}\right] \otimes 1\right) \oplus\left(x_{j}^{d-1} \mathbb{C}\left[x_{j}^{d}\right] \otimes x_{j}\right) & \text { if } i=0 \\ \left(x_{j}^{d-i} \mathbb{C}\left[x_{j}^{d}\right] \otimes 1\right) \oplus\left(x_{j}^{d-1-i} \mathbb{C}\left[x_{j}^{d}\right] \otimes x_{j}\right) & \text { if } 1 \leq i \leq d-1\end{cases}
$$

The $i=0$ case. The space $\left(\mathbb{C}\left[x_{j}^{d}\right] \otimes 1\right) \oplus\left(x_{j}^{d-1} \mathbb{C}\left[x_{j}^{d}\right] \otimes x_{j}\right)$ is the tensor product of a symmetric algebra $\mathbb{C}\left[x_{j}^{d}\right]$ on the generator $x_{j}^{d}$ with an exterior algebra on the generator $x_{j}^{d-1} \otimes x_{j}$. Consequently, the $(\mathbb{Z} / d \mathbb{Z})^{n}-$ fixed space (3.14) is a symmetric algebra $\mathbb{C}\left[x_{1}^{d}, \ldots, x_{n}^{d}\right]$ tensored with the exterior algebra on the generators $\left\{x_{j}^{d-1} \otimes x_{j}\right\}_{j=1}^{n}$. Since the residual action of $\mathfrak{S}_{n}$ permutes the subscripts as usual, one concludes that the last expression in (3.13) is $P_{\mathfrak{S}_{n}}\left(\chi^{\lambda} ; q^{d}, t q^{d-1}\right)$ for $i=0$.

The $1 \leq i \leq d-1$ case. We proceed as in the $i=0$ case. First enlarge coefficients from $\mathbb{C}\left[x_{i}\right]$ to Frac $\left(\mathbb{C}\left[x_{i}\right]\right)$. Then $\left(x_{j}^{d-i} \mathbb{C}\left[x_{j}^{d}\right] \otimes 1\right) \oplus\left(x_{j}^{d-1-i} \mathbb{C}\left[x_{j}^{d}\right] \otimes x_{j}\right)$ is a free module of rank 1 with basis element $x_{j}^{d-i}$ over the subalgebra of $\operatorname{Frac}\left(\mathbb{C}\left[x_{i}\right]\right) \otimes \wedge \mathbb{C} x_{j}$ which is the tensor product of a symmetric algebra $\mathbb{C}\left[x_{j}^{d}\right]$ on generator $x_{j}^{d}$ with an exterior algebra on generator $x_{j}^{-1} \otimes x_{j}$. Consequently, the $(\mathbb{Z} / d \mathbb{Z})^{n}$-fixed space (3.14) is a free module of rank 1 with basis element $\left(x_{1} \cdots x_{n}\right)^{d-i}$ over the subalgebra of $\operatorname{Frac}(S) \otimes \wedge V^{*}$ given as the tensor product of the symmetric algebra $\mathbb{C}\left[x_{1}^{d}, \ldots, x_{n}^{d}\right]$ with the exterior algebra on the generators $\left\{x_{j}^{-1} \otimes x_{j}\right\}_{j=1}^{n}$. Since $\mathfrak{S}_{n}$ still permutes the subscripts, the last expression in (3.13) is just $q^{n(d-i)} P_{\mathfrak{S}_{n}}\left(\chi^{\lambda} ; q^{d}, t q^{-1}\right)$.

We substitute these expressions for each $i$ in (3.12) to obtain the product on the right in the proposition.

Remark 3.15. For the sake of the reader wishing to compare notation with that of Koike 20], note that the group we call $W_{n}=G(d, 1, n)$ is his group $G_{n, d}$, and the group that we call $G(d e, e, n)$ below is his group $G_{n, d, e}$. Also, because he works with $S(V) \otimes \wedge(V)$ rather than $S\left(V^{*}\right) \otimes \wedge\left(V^{*}\right)$, his Theorem 1 calculates what we denote here by $P_{W_{n}}\left(\left(\chi^{\underline{\lambda}}\right)^{*} ; q, t\right)$, where one can identify the contragredient representation of $\chi^{\underline{\lambda}}=\chi^{\left(\lambda^{(0)}, \lambda^{(1)}, \ldots, \lambda^{(r-1)}\right)}$ with $\left(\chi^{\underline{\lambda}}\right)^{*}=\chi^{\left(\lambda^{(0)}, \lambda^{(r-1)}, \ldots, \lambda^{(2)}, \lambda^{(1)}\right)}$.

Corollary 3.16. The conclusion of Theorem 1.1 holds for the Shephard-Todd family $G(d, 1, n)$ with $d \geq 2$.

Proof. According to (3.2), we should compute $P_{W}\left(\left(\chi_{\wedge^{r} V}\right)^{*} ; q, t\right)$ for $W=G(d, 1, n)$ acting on $V=\mathbb{C}^{n}$ and $0 \leq r \leq n$. One can check that $\left(\chi_{\wedge^{r} V}\right)^{*}$ is the $W$-irreducible character $\chi^{\underline{\lambda}}$ for $\underline{\lambda}=\left(\lambda^{(0)}, \ldots, \lambda^{(d-1)}\right)$ where

$$
\lambda^{(0)}=(n-r), \quad \lambda^{(1)}=\lambda^{(2)}=\cdots=\lambda^{(d-2)}=\varnothing, \quad \text { and } \quad \lambda^{(d-1)}=\left(1^{r}\right) .
$$

Hence Theorem 3.11 together with Corollary 3.4 gives

$$
\begin{aligned}
P_{G(d, 1, n)}\left(\left(\chi_{\wedge r}\right)^{*} ; q, t\right) & =P_{\mathfrak{S}_{n-r}}\left(\chi^{(n-r)} ; q^{d}, t q^{d-1}\right) q^{r} P_{\mathfrak{S}_{r}}\left(\chi^{\left(1^{r}\right)} ; q^{d}, t q^{-1}\right) \\
& =\prod_{x \in(n-r)} \frac{1+t q^{d-1+d c(x)}}{1-q^{d h(x)}} q^{r} q^{d\left(\begin{array}{c}
r \\
2
\end{array}\right)} \prod_{x \in\left(1^{r}\right)} \frac{1+t q^{-1+d c(x)}}{1-q^{d h(x)}} \\
& =q^{r+d\left(\begin{array}{c}
r \\
2
\end{array}\right)} \prod_{i=1}^{n-r} \frac{1+t q^{d-1+d(i-1)}}{1-q^{d i}} \prod_{i=1}^{r} \frac{1+t q^{-1-d(i-1)}}{1-q^{d i}} \\
& =q^{r+d\left(\begin{array}{c}
r \\
2
\end{array}\right)}\left[\begin{array}{l}
n \\
r
\end{array}\right]_{q^{d}} \frac{\left(-t q^{d-1} ; q^{d}\right)_{n-r}\left(-t q^{-1} ; q^{-d}\right)_{n}}{\left(q^{d} ; q^{d}\right)_{n}}
\end{aligned}
$$


using (1.2) and (1.3) in the second and third equalities. As $G(d, 1, n)$ has rank $n$ and exponents

$$
\left(e_{1}, \ldots, e_{n}\right)=(d-1,2 d-1, \ldots, n d-1),
$$

its smallest exponent is $e_{1}=d-1$ and the gap between exponents is $a=d$. Hence this last expression agrees with that in Theorem 1.1, which is equivalent to Theorem 1.1.

Koike's formula for $G(d e, e, n)$. Koike also generalized Theorem 3.11 from the wreath product groups $G(d, 1, n)$ to the entire infinite family $G(d e, e, n)$ in Shephard and Todd's classification of irreducible complex reflection groups 31. Here $G(d e, e, n)$ for positive integers $d, e, n$ is the the kernel of the degree one character $\epsilon^{d}: G(d e, 1, n) \rightarrow \mathbb{C}^{\times}$, where $\epsilon$ was defined in (3.10). In other words, $G(d e, e, n)$ is the group of monomial $n \times n$ matrices whose nonzero entries are all $(d e)^{t h}$ roots-of-unity and for which the product of the nonzero entries is a $d^{t h}$ root-of-unity. Although the group $G(d e, e, n)$ is only coincidental when $e=1$, we find it worthwhile to state his result and then use it to compute the Hilbert series of $\left(S \otimes \wedge V^{*} \otimes \wedge^{r} V\right)^{G(d e, e, n)}$, so that we can see how it would differ from the form in Theorem 1.1 when $e \geq 2$.

First we recall the parametrization of irreducible $G(d e, e, n)$-representations; see, e.g., [20, §2]. Given an irreducible $G(d e, 1, n)$-character $\chi^{\underline{\lambda}}$ corresponding to a multipartition $\underline{\lambda}=\left(\lambda^{(0)}, \lambda^{(1)}, \ldots, \lambda^{(d e-1)}\right)$ of $n$, consider the superscripts $i$ in $\lambda^{(i)}$ as taken modulo $d e$, so that $\lambda^{(i+d e)}=\lambda^{(i)}$ for all integers $i$. Then the restriction of $\chi^{\underline{\lambda}}$ from $G(d e, 1, n)$ to $G(d e, e, n)$ depends only upon the orbit of $\underline{\lambda}$ under the operation that replaces $\lambda^{(i)}$ by $\lambda^{(i+d)}$ for all $i$. If one fixes a representative $\underline{\lambda}$ of this orbit and defines a positive integer

$$
\mu(\underline{\lambda}):=\min \left\{m \geq 1: \lambda^{(i)}=\lambda^{(i+d m)} \text { for all } i\right\},
$$

then $\chi^{\underline{\lambda}}$ decomposes upon restriction to $G(d e, e, n)$ into $e / \mu(\underline{\lambda})$ inequivalent $G(d e, e, n)$-irreducible characters, and each $G(d e, e, n)$-irreducible arises once in this way. Koike's result may then be stated as follows.

Theorem 3.17. [20, Theorem 2] For a multipartition $\underline{\lambda}$ of $n$ parametrizing an irreducible character $\chi^{\underline{\lambda}}$ of $G(d e, 1, n)$, let $\hat{\chi}^{\underline{\lambda}}$ denote any $G(d e, e, n)$-irreducible constituent of the restriction to $G(d e, e, n)$. Then

$$
P_{G(d e, e, n)}\left(\hat{\chi}^{\underline{\lambda}} ; q, t\right)=\sum_{v=0}^{\mu(\underline{\lambda})-1} P_{\mathfrak{S}_{n_{d v}}}\left(\chi^{\lambda^{(d v)}} ; q^{d e}, t q^{d e-1}\right) \cdot \prod_{i=1}^{d e-1} q^{n_{d v+i} \cdot(d e-i)} \cdot P_{\mathfrak{S}_{n_{i+d v}}}\left(\chi^{\lambda^{(i+d v)}} ; q^{d e}, t q^{-1}\right) .
$$

In particular, the answer is independent of the chosen irreducible constituent $\hat{\chi}^{\underline{\underline{\lambda}}}$ and depends only on $\chi^{\underline{\lambda}}$.

Note that for $e=1$, every $\underline{\lambda}$ has $\mu(\underline{\lambda})=1$, so that the sum has only the $v=0$ term, recovering Theorem 3.11

Corollary 3.18. Let $W=G(d e, e, n)$ with $d, e \geq 1$ and $n, d e \geq 2$. Then the various Hilbert series

$$
\operatorname{Hilb}\left(\left(S \otimes \wedge V^{*} \otimes \wedge^{r} V\right)^{W}, q, t\right)=P_{W}\left(\left(\chi_{\wedge^{r} V}\right)^{*} ; q, t\right)
$$

for $0 \leq r \leq n$ have these formulas:

- When $r=0$, it is

$$
\frac{\left(-t q^{d e-1} ; q^{d e}\right)_{n-1}\left(1+t q^{d n-1}\right)}{\left(q^{d e} ; q^{d e}\right)_{n-1}\left(1-q^{d n}\right)} .
$$

- When $1 \leq r \leq n-1$ and $d \geq 2$, it is

$$
q^{d e\left(\begin{array}{l}
r \\
2
\end{array}\right)+r} \frac{\left(-t q^{-1} ; q^{-d e}\right)_{r}\left(-t q^{d e-1} ; q^{d e}\right)_{n-1-r}}{\left(q^{d e} ; q^{d e}\right)_{r}\left(q^{d e} ; q^{d e}\right)_{n-r}\left(1-q^{d n}\right)}\left(1-q^{d e n}+t q^{-1}\left(q^{d e(n-r)}\left(1-q^{d n}\right)+q^{d n}-q^{d e n}\right)\right) .
$$

- When $1 \leq r \leq n-1$ and $d=1$, it is

$$
\begin{aligned}
& q^{e\left(\begin{array}{c}
r \\
2
\end{array}\right)} \frac{\left(-t q^{-1} ; q^{-e}\right)_{r-1}\left(-t q^{e-1} ; q^{e}\right)_{n-1-r}}{\left(q^{e} ; q^{e}\right)_{r}\left(q^{e} ; q^{e}\right)_{n-r}\left(1-q^{n}\right)}\left(q^{r}\left(1+t q^{(n-r) e-1}\right)\left(1+t q^{-(r-1) e-1}\right)\left(1-q^{n}\right)\right. \\
& +q^{(n-r)(e-1)-1}\left(1+t q^{e-1}\right)\left(1-q^{n}\right)(q+t) \\
& \left.+q^{r-1}\left(1+t q^{-(r-1) e-1}\right)(q+t)\left(q^{n}-q^{n(e-1)}\right)\right) \text {. }
\end{aligned}
$$

- When $r=n$, it is

$$
q^{d e\left(\begin{array}{c}
n-1 \\
2
\end{array}\right)+n-1} \frac{\left(-t q^{-1} ; q^{-d e}\right)_{n-1}}{\left(q^{d e} ; q^{d e}\right)_{n-1}\left(1-q^{d n}\right)} \cdot \begin{cases}\left(q^{d e(n-1)+1}+t\right) & \text { if } d \geq 2 \\
\left(q^{(n-1)(e-1)}+t\right) & \text { if } d=1\end{cases}
$$


Proof. We only sketch the somewhat tedious proofs, which are of the same nature as those for Corollary 3.16

The $G(d e, e, n)$-representation $\left(\wedge^{r} V\right)^{*}$ is the restriction of the $G(d e, 1, n)$-representation $\left(\wedge^{r} V\right)^{*}$, and the latter has character $\chi^{\underline{\lambda}}$ where $\underline{\lambda}=\left((n-r), \varnothing, \varnothing, \ldots, \varnothing,\left(1^{r}\right)\right)$. Thus one can apply Theorem 3.17 to compute $P_{G(d e, e, n)}\left(\hat{\chi}^{\underline{\lambda}} ; q, t\right)$. In this case, $\mu(\underline{\lambda})=e$, and so the sum in Theorem 3.17 always has $e$ terms.

When $r=0$, since $\underline{\lambda}$ has only one nonempty component $\lambda^{(0)}=(n)$, each summand in Theorem 3.17 for $v=0,1,2, \ldots, e-1$ has only one non-unit factor: the $v=0$ summand is the factor $P_{\mathfrak{S}_{n}}\left(\chi^{(n)} ; q^{d e}, t q^{d e-1}\right)$, and each of the $v=1,2, \ldots, e-1$ summands is the factor indexed by $i=d(e-v)$ in the product. After pulling out common factors, one can sum the geometric series over $v=1,2, \ldots, e-1$, and some simplification then leads to the formula given in the corollary. The proof for $r=n$ is extremely similar.

When $1 \leq r \leq n-1$, since $\underline{\lambda}$ has two nonempty components $\lambda^{(0)}=(n-r)$ and $\lambda^{(d e-1)}=\left(1^{r}\right)$, each summand for Theorem 3.17 for $v=0,1,2, \ldots, e-1$ is a product of two non-unit factors.

- The $v=0$ summand is the factor in front of the product times the $i=1$ factor within the product.

- If $d=1$, the $v=1$ summand is similarly the factor in front times the factor indexed by $i=e-1$ within the product, while each of the $v=2,3, \ldots, e-1$ summands is the product of the two factors for $i=e-v, e-v+1$ in the product.

- If $d \geq 2$ so that $e-1<d e-1$, each of the $v=1,2, \ldots, e-1$ summands is the product of the two factors for $i=d(e-v), d(e-v)+1$ in the product.

In each case, one pulls out common factors, sums a geometric series, and simplifies to obtain the formula.

Remark 3.19. One can compare the formulas for the Hilbert series in Corollary 3.18 to see how they differ from the formulas in Theorem 1.1. Note that $G(d e, e, n)$ has

$$
\begin{aligned}
\left(d_{1}, d_{2}, \ldots, d_{n}\right) & =(d e, 2 d e, \ldots,(n-1) d e, d n), \\
\left(e_{1}, e_{2}, \ldots, e_{n}\right) & =(d e-1,2 d e-1, \ldots,(n-1) d e-1, d n-1), \\
\left(e_{1}^{*}, e_{2}^{*}, \ldots, e_{n}^{*}\right) & = \begin{cases}(1,1+d e, 1+2 d e, \ldots, 1+(n-2) d e, 1+(n-1) d e) & \text { if } d \geq 2, \\
(1,1+e, 1+2 e, \ldots, 1+(n-2) e,(n-1)(e-1)) & \text { if } d=1 .\end{cases}
\end{aligned}
$$

It is then not hard to check that the cases $r=0$ and $r=n$ of Corollary 3.18 agree with the formula given in Theorem 1.1] as predicted by Solomon's formula (2.6) for $r=0$ and Theorem 2.10 for $r=n$.

For $1 \leq r \leq n-1$, one can check using (3.20) that the formula in Theorem 1.1 would assert for $d \geq 2$ that

$$
\operatorname{Hilb}\left(\left(S \otimes \wedge V^{*} \otimes \wedge^{r} V\right)^{W}, q, t\right)=q^{d e\left(\begin{array}{c}
r \\
2
\end{array}\right)+r} \frac{\left(-t q^{-1} ; q^{-d e}\right)_{r}\left(-t q^{d e-1} ; q^{d e}\right)_{n-1-r}\left(1+t q^{d \min \{(n-r) e, n\}-1}\right)}{\left(q^{d e} ; q^{d e}\right)_{r}\left(q^{d e} ; q^{d e}\right)_{n-r}} .
$$

In general, this formula is incorrect - one can check that it disagrees with the corresponding expression in Corollary 3.18 except when $e=1$, namely when $G(d e, e, n)=G(d, 1, n)$, a coincidental group.

Similarly, for $d=1$, so that $W=G(e, e, n)$, one can check using (3.20) that the formula in Theorem 1.1 would assert that

$$
\begin{aligned}
& \operatorname{Hilb}\left(\left(S \otimes \wedge V^{*} \otimes \wedge^{r} V\right)^{W}, q, t\right) \\
& \begin{aligned}
=q^{e\left(\frac{r}{2}\right)+r} \cdot \frac{\left(-t q^{-1} ; q^{-e}\right)_{r-1}\left(-t q^{e-1} ; q^{e}\right)_{n-1-r}}{\left(q^{e} ; q^{e}\right)_{r-1}\left(q^{e} ; q^{e}\right)_{n-r}} \\
\quad \cdot \frac{\left(1+t q^{\min \{(n-r) e, n\}-1}\right)\left(1+t q^{-\min \{1+(r-1) e,(n-1)(e-1)\}}\right)}{\left(1-q^{r e}\right)\left(1-q^{n}\right)} \cdot\left(1-q^{n e-r e}+q^{n e-r e-n}-q^{n e-n}\right) .
\end{aligned}
\end{aligned}
$$

In general, this formula is also incorrect - one can check it disagrees with the $d=1$ case of Corollary 3.18 except when $r=1$, in which case both formulas agree with (2.9) since $G(e, e, n)$ is a duality group.

\section{Conjectured explicit Basis}

Here we strengthen Theorem 1.1 to conjecture an explicit basis of the space of invariants $\left(S \otimes \wedge V^{*} \otimes \wedge V\right)^{W}$, extending ideas of [33. In fact, for all of the remaining coincidental groups $W$ (not type $A$ nor $G(d, 1, n)$ ), we verify this stronger conjecture in later sections to complete the verification of Theorem [1.1] Let us view

$$
M_{r}:=\left(S\left(V^{*}\right) \otimes \wedge V^{*} \otimes \wedge^{r} V\right)^{W}
$$


as a module over the exterior algebra $\left(S\left(V^{*}\right) \otimes \wedge V^{*}\right)^{W}=\wedge_{S\left(V^{*}\right)^{W}}\left\{d f_{1}, \ldots, d f_{n}\right\}$ via multiplication into the first two tensor factors. We require notation for letting a derivation act as an operator on $M_{r}$ by taking partial derivatives of polynomial coefficients: Given any derivation $\theta=\sum_{j=1}^{n} h_{j}\left(x_{1}, \ldots, x_{n}\right) \otimes y_{j}$ in $S\left(V^{*}\right) \otimes V$, let

$$
\tilde{\theta}: S\left(V^{*}\right) \otimes \wedge^{k} V^{*} \otimes \wedge V \longrightarrow S\left(V^{*}\right) \otimes \wedge^{k+1} V^{*} \otimes \wedge V
$$

be the differential operator defined by

$$
f \otimes \eta \otimes \eta^{\prime} \quad \longmapsto \quad \sum_{j=1}^{n} \bar{h}_{j}\left(\frac{\partial}{\partial x_{1}}, \ldots, \frac{\partial}{\partial x_{n}}\right)(f) \otimes x_{j} \wedge \eta \otimes \eta^{\prime},
$$

with bar indicating that complex coefficients are conjugated. Here, each $x_{i}^{m}$ in the polynomial $h_{j}$ is replaced by the iterated partial derivative $\partial^{m} / \partial x_{i}^{m}$ and the resulting operator is applied to $f$.

Note that in the case where $\theta$ is the Euler derivation $\theta_{E}=\sum_{j=1}^{n} x_{i} \otimes y_{j}$ in $S\left(V^{*}\right) \otimes V$, the operator $\tilde{\theta}_{E}$ restricts to the the usual exterior derivative $d: S\left(V^{*}\right) \mapsto S\left(V^{*}\right) \otimes V^{*}$. Restricting $\tilde{\theta}_{E}$ merely to a map $S\left(V^{*}\right) \otimes \wedge^{0} V^{*} \otimes V \rightarrow S \otimes \wedge^{1} V^{*} \otimes V$ gives the operator $\psi \mapsto d \psi$ from [28. We can now state our conjecture.

Conjecture 4.1. For $W$ a coincidental reflection group, one may choose basic invarints $f_{1}, \ldots, f_{n}$ and basic derivations $\theta_{1}, \ldots, \theta_{n}$ so that each $M_{r}=\left(S\left(V^{*}\right) \otimes \wedge V^{*} \otimes \wedge^{r} V\right)^{W}$ for $r=1, \ldots, n$ is a free module over the exterior algebra $R_{r}:=\wedge_{S\left(V^{*}\right)^{W}}\left\{d f_{1}, \ldots, d f_{n-r}\right\}$ with basis

$$
\tilde{\theta}_{i_{1}} \cdots \tilde{\theta}_{i_{m}}\left(\theta_{j_{1}} \wedge \cdots \wedge \theta_{j_{r}}\right)
$$

for $0 \leq m \leq r$ and $1 \leq i_{1}<\cdots<i_{m} \leq r$ and $1 \leq j_{1}<\cdots<j_{r} \leq n$. For $r=0$, take basis element 1 .

Equivalently one can write the conjectured basis more directly in terms of subsets of $\{1, \ldots, n\}$. Define

$$
\begin{array}{rlrl}
\tilde{\theta}_{I} & :=\tilde{\theta}_{i_{1}} \cdots \tilde{\theta}_{i_{m}}: S\left(V^{*}\right) \otimes \wedge^{k} V^{*} \otimes \wedge V \longrightarrow S\left(V^{*}\right) \otimes \wedge^{k+m} V^{*} \otimes \wedge V & & \text { for } I=\left\{i_{1}<\cdots<i_{m}\right\}, \\
\theta_{J} & :=\theta_{j_{1}} \wedge \cdots \wedge \theta_{j_{r}} \quad \text { in } S\left(V^{*}\right) \otimes \wedge^{0} \otimes \wedge^{r} V & \text { for } J=\left\{j_{1}<\cdots<j_{r}\right\}, \\
d f_{L}: & =d f_{\ell_{1}} \wedge \cdots \wedge d f_{\ell_{m^{\prime}}} \text { in } S\left(V^{*}\right) \otimes \wedge^{m^{\prime}} V^{*} \otimes \wedge^{0} V & \text { for } L=\left\{\ell_{1}<\cdots<\ell_{m^{\prime}}\right\} .
\end{array}
$$

Conjecture 4.1 is then equivalent to the following statement.

Conjecture 4.1. For $W$ a coincidental reflection group, one may choose basic invariants $f_{1}, \ldots, f_{n}$ and basic derivations $\theta_{1}, \ldots, \theta_{n}$ so that

$$
M_{r, k}:=\left(S\left(V^{*}\right) \otimes \wedge^{k} V^{*} \otimes \wedge^{r} V\right)^{W} \quad \text { for } \quad 1 \leq r, k \leq n
$$

is a free module over $S\left(V^{*}\right)^{W}$ with basis

$$
\left\{d f_{L} \cdot \tilde{\theta}_{I}\left(\theta_{J}\right)\right\}
$$

as one runs through all triples $(I, J, L)$ of subsets $J \subset[n], I \subset[r], L \subset[n-r]$ with $|J|=r$ and $|I|+|L|=k$.

Remark 4.3. Note that several special cases of the equivalent Conjectures 4.1 and 4.1 hold more generally:

- Conjecture 4.1 at $r=0$ holds for all reflection groups by [35].

- Conjecture 4.1 at $r=1$ holds for all duality groups by [28, Thm 1.1].

- Conjecture 4.1 at $k=0$ holds for all reflection groups by [25, Thm. 3.1].

Proposition 4.4. Conjecture 4.1] (or its equivalent Conjecture 4.1) implies Theorem 1.1].

Proof. The conjecture gives an $S^{W}$-basis for $\left(S \otimes \wedge V^{*} \otimes \wedge^{r} V\right)^{W}$ where we again appreviate $S=S\left(V^{*}\right)$; we compare the polynomial degrees of basis elements in the conjecture with the coefficient of $s^{r}$ on the right in Theorem 1.1. Since $\operatorname{Hilb}\left(S^{W}, q\right)=\prod_{i=1}^{n}\left(1-q^{d_{i}}\right)^{-1}$, after clearing the denominator in Theorem 1.1. we have

$$
\left(\prod_{\ell=1}^{n-r}\left(1+q^{e_{\ell}} t\right)\right)\left(\prod_{i=1}^{r}\left(1+q^{-e_{i}^{*}} t\right)\right) \sigma_{r}\left(q^{e_{1} *}, \ldots, q^{e_{n}^{*}}\right) .
$$


We check that this matches the $(q, t)$-bidegrees of the $S^{W}$-basis elements $\left\{d f_{L} \cdot \tilde{\theta}_{I}\left(\theta_{J}\right)\right\}$ in Conjecture 4.1]:

$$
\begin{aligned}
& \left(\prod_{\ell=1}^{n-r}\left(1+q^{e_{\ell}} t\right)\right)\left(\prod_{i=1}^{r}\left(1+q^{-e_{i}^{*}} t\right)\right) \sigma_{r}\left(q^{e_{1} *}, \ldots, q^{e_{n}^{*}}\right) \\
& =\left(\sum_{L \subset[n-r]} t^{|L|} q^{\sum_{\ell \in L} e_{\ell}}\right)\left(\sum_{I \subset[r]} t^{|I|} q^{-\sum_{i \in I} e_{i}^{*}}\right)\left(\sum_{\substack{J \subset[n]: \\
|J|=r}} q^{\sum_{j \in J} e_{j}^{*}}\right)=\sum_{(I, J, L)} t^{|I|+|L|} q^{\sum_{\ell \in L} e_{\ell}+\sum_{j \in J} e_{j}^{*}-\sum_{i \in I} e_{i}^{*}},
\end{aligned}
$$

where the last sum runs through $(I, J, L)$ satisfying the conditions in Conjecture 4.1 . Theorem 1.1 then follows since $d f_{L} \cdot \tilde{\theta}_{I}\left(\theta_{J}\right)$ has $\wedge V^{*}$-degree $|I|+|L|$ and $S$-degree $\sum_{\ell \in L} e_{\ell}+\sum_{j \in J} e_{j}^{*}-\sum_{i \in I} e_{i}^{*}$.

\section{The elements in Conjecture 4.1 ARE InVARIANT}

As a precursor to verifying Conjecture 4.1 or 4.1 , we check that the forms indicated there are indeed invariant under the action of any reflection group $W$.

Lemma 5.1. For $V=\mathbb{C}^{n}$, let $W \subset G L(V)$ be a group of isometries and let $S=S\left(V^{*}\right)$.

(a) The map $(S \otimes V \otimes 1) \times\left(S \otimes \wedge^{k} V^{*} \otimes \wedge^{r} V\right) \rightarrow S \otimes \wedge^{k+1} V^{*} \otimes \wedge^{r} V, \quad(\theta, \omega) \mapsto \widetilde{\theta}(\omega)$, is $W$-equivariant.

(b) For $\theta$ in $(S \otimes 1 \otimes V)^{W}$ and $\omega$ in $\left(S \otimes \wedge^{k} V^{*} \otimes \wedge^{r} V\right)^{W}$, the form $\tilde{\theta}(\omega)$ lies in $\left(S \otimes \wedge^{k+1} V^{*} \otimes \wedge^{r} V\right)^{W}$.

Proof. For any polynomials $h, f$ in $S$, let $\partial(h)(f):=\bar{h}\left(\frac{\partial}{\partial x_{1}}, \ldots, \frac{\partial}{\partial x_{n}}\right)(f)$. The map $S \times S \rightarrow S$ given by $(h, f) \mapsto \partial(h)(f)$ is $W$-equivariant (see [33, $\S 6])$ : For $g$ in $W$,

$$
g(\partial(h) f)=\partial(g h)(g f) .
$$

Now consider some $\theta=h \otimes 1 \otimes y_{j}$ in $S \otimes 1 \otimes V$ and $\omega=f \otimes \eta \otimes \eta^{\prime}$ in $S \otimes \wedge V^{*} \otimes \wedge V$. For any $g$ in $W$, $(g \theta, g \omega)$ maps to $g(\tilde{\theta}(\omega))$ under the function in (a) since

$$
\begin{aligned}
g(\tilde{\theta}(\omega)) & =g\left(\partial(h) f \otimes x_{j} \wedge \eta \otimes \eta^{\prime}\right)=g(\partial(h) f) \otimes g x_{j} \wedge g \eta \otimes g \eta^{\prime} \\
& =\partial(g(h))(g f) \otimes g\left(x_{j} \wedge \eta\right) \otimes g \eta^{\prime}=\widetilde{g \theta}\left(g\left(f \otimes \eta \otimes \eta^{\prime}\right)\right)=\widetilde{g \theta}(g \omega) .
\end{aligned}
$$

Thus $(\theta, g \omega)$ maps to $g(\tilde{\theta}(\omega))$ whenever $\theta$ is $W$-invariant, and $\tilde{\theta}(\omega)$ is $W$-invariant whenever $\theta$ and $\omega$ are.

Remark 5.2. Recall that for any linear transformation $g$ in $\mathrm{GL}(V)$, the matrix recording the dual action of $g$ on $V^{*}$ with respect to a dual basis is just the inverse transpose of the matrix recording the action of $g$ on $V$. As $W$ is finite, we may and have assumed the matrices giving the action of $W$ are all unitary, so that the matrix of $g$ in $W$ acting on $V^{*}$ is the complex conjugate (entry-wise) of the matrix of $g$ acting on $V$. This explains why we take the complex conjugates of coefficients in defining the operators $\tilde{\theta}$.

\section{Conjecture 4.1 agrees with the Gutkin-Opdam calculation}

As a second precursor to verifying Conjecture 4.1 (or 4.1), we check that it correctly predicts the sum of the degrees of the homogeneous elements in a basis for $\left(S \otimes \wedge^{k} V^{*} \otimes \wedge^{r} V\right)^{W}$ over $S\left(V^{*}\right)^{G}$ when $W$ is a duality group. For any reflection group $W$, set (e.g., see [5, §4.5.5, Remark 4.48]))

$$
\begin{aligned}
N & :=\#\{\text { reflections in } W\}=\sum_{i=1}^{n} e_{i}, \\
N^{*} & :=\#\{\text { reflecting hyperplanes for } W\}=\sum_{i=1}^{n} e_{i}^{*} .
\end{aligned}
$$

Recall that for any $W$-representation $U$, the sum of the $U$-exponents

$$
\psi(U)=e_{1}(U)+\ldots+e_{\operatorname{dim} U}(U)
$$

is the sum of the polynomial degrees of homogeneous elements in any basis of $\left(S\left(V^{*}\right) \otimes U\right)^{W}$ as a free module over $S\left(V^{*}\right)^{W}$. The Gutkin-Opdam Lemma [5, Prop. 4.3.3, eqn. (4.6)] allows one to predict $\psi(U)$ as follows:

$$
\begin{aligned}
\psi(U) & =\sum_{H} \sum_{j=0}^{e_{H}-1} j \cdot\left\langle U \downarrow_{W_{H}}^{W}, \operatorname{det}^{j}\right\rangle_{W_{H}} \\
& =\sum_{H} D_{H}\left(U \downarrow_{W_{H}}^{W}\right),
\end{aligned}
$$


where $H$ runs through the reflecting hyperplanes for $W$, with $W_{H} \subset W$ the pointwise stabilizer subgroup of $H$ and $e_{H}:=\left|W_{H}\right|$. Here $D_{H}$ is the linear functional on the Grothendieck group $G_{0}\left(W_{H}\right)$ of $W_{H}$-representations that sends the 1 -dimensional representation $\operatorname{det}^{j}$ to $j$ for $j=0,1, \ldots, e_{H}-1$.

Lemma 6.2. Let $W$ be a reflection group on $V$ and $U=\wedge^{k} V^{*} \otimes \wedge^{r} V$. Then

$$
\psi(U)=\left(\begin{array}{c}
n-1 \\
k-1
\end{array}\right)\left(\begin{array}{c}
n-1 \\
r
\end{array}\right) N+\left(\begin{array}{c}
n-1 \\
k
\end{array}\right)\left(\begin{array}{l}
n-1 \\
r-1
\end{array}\right) N^{*} .
$$

Proof. Let $H$ be a reflecting hyperplane of $W$. The restrictions of $V^{*}$ or $V$ to $W_{H}$ each contain $n-1$ copies of the trivial $W_{H}$-representation as direct summands, and then one extra summand carrying the 1-dimensional representations $\operatorname{det}^{e_{H}-1}$ or det, respectively. We sum over all $k, r$, apply the Gutkin-Opdam Lemma, and then extend $D_{H}$ to be linear over $\mathbb{C}[s, t]$ keeping (6.1) in mind:

$$
\begin{aligned}
\sum_{k, r} \psi\left(\wedge^{k} V^{*} \otimes \wedge^{r} V\right) t^{k} s^{r} & =\sum_{H} \sum_{k, r} D_{H}\left(\wedge^{k} V^{*} \otimes \wedge^{r} V \downarrow_{W_{H}}^{W}\right) t^{k} s^{r} \\
& =\sum_{H} D_{H}\left((1+t)^{n-1}\left(1+t \operatorname{det}^{e_{H}-1}\right)(1+s)^{n-1}(1+s \operatorname{det})\right) \\
& =(1+t)^{n-1}(1+s)^{n-1} \sum_{H} D_{H}\left(\left(1+t \operatorname{det}^{e_{H}-1}\right)(1+s \operatorname{det})\right) \\
& =(1+t)^{n-1}(1+s)^{n-1} \sum_{H} D_{H}\left(\left(1+t \operatorname{det}^{e_{H}-1}+s \operatorname{det}+s t\right)\right) \\
& =(1+t)^{n-1}(1+s)^{n-1} \sum_{H}\left(t\left(e_{H}-1\right)+s\right) \\
& =(1+t)^{n-1}(1+s)^{n-1}\left(t N+s N^{*}\right) .
\end{aligned}
$$

The result then follows from extracting the coefficient of $t^{k} s^{r}$.

Proposition 6.3. Either of Theorem 1.1 or Conjecture 4.1 correctly predicts $\psi(U)$ for each $U=\wedge^{k} V^{*} \otimes \wedge^{r} V$ with $0 \leq k, r \leq n$ for any irreducible duality reflection group $W$.

Proof of Proposition 6.3. On one hand, $\sum_{k, r} \psi(U) t^{k} s^{r}=(1+t)^{n-1}(1+s)^{n-1}\left(t N+s N^{*}\right)$ by Lemma 6.2 We fix $r=0,1, \ldots, n$ and extract the coefficient of $s^{r}$ to obtain

$$
\sum_{k} \psi\left(\wedge^{k} V^{*} \otimes \wedge^{r} V\right) t^{k}=(1+t)^{n-1}\left(t N\left(\begin{array}{c}
n-1 \\
r
\end{array}\right)+N^{*}\left(\begin{array}{c}
n-1 \\
r-1
\end{array}\right)\right) .
$$

On the other hand, Theorem 1.1 predicts for each $r=0, \ldots, n$ that

$$
\begin{aligned}
\sum_{k} \psi\left(\wedge^{k} V^{*} \otimes \wedge^{r} V\right) t^{k} & =\lim _{q \rightarrow 1}\left[\frac{\partial}{\partial q}\left(\sigma_{r}\left(q^{e_{1}^{*}}, \ldots, q^{e_{n}^{*}}\right) \cdot \prod_{i=1}^{n-r}\left(1+q^{e_{i}} t\right) \cdot \prod_{i=1}^{r}\left(1+q^{-e_{i}^{*}} t\right)\right)\right] \\
& =\sum_{\substack{J \subset\{1,2, \ldots, n\}: \\
|J|=r}} \lim _{q \rightarrow 1}\left[\frac{\partial}{\partial q}\left(q^{\sum_{j \in J}} e_{j}^{*} \cdot \prod_{i=1}^{n-r}\left(1+q^{e_{i}} t\right) \cdot \prod_{i=1}^{r}\left(1+q^{-e_{i}^{*}} t\right)\right)\right] \\
& =\sum_{\substack{J \subset\{1,2, \ldots, n\}: \\
|J|=r}}\left[(1+t)^{n} \sum_{j \in J} e_{j}^{*}+(1+t)^{n-1} \sum_{i=1}^{n-r} t e_{i}+(1+t)^{n-1} \sum_{i=1}^{r}\left(-t e_{i}^{*}\right)\right] \\
& =(1+t)^{n-1}\left[\sum_{i=1}^{n} e_{i}^{*}(1+t)\left(\begin{array}{c}
n-1 \\
r-1
\end{array}\right)+t\left(\begin{array}{c}
n \\
r
\end{array}\right)\left(\sum_{i=1}^{n-r} e_{i}-\sum_{i=1}^{r} e_{i}^{*}\right)\right]
\end{aligned}
$$


It only remains to check the bracketed expression is $t N\left(\begin{array}{c}n-1 \\ r\end{array}\right)+N^{*}\left(\begin{array}{c}n-1 \\ r-1\end{array}\right)$. We use (6.1):

$$
\begin{aligned}
\sum_{i=1}^{n} e_{i}^{*}(1+t) & \left(\begin{array}{l}
n-1 \\
r-1
\end{array}\right)+t\left(\begin{array}{l}
n \\
r
\end{array}\right)\left(\sum_{i=1}^{n-r} e_{i}-\sum_{i=1}^{r} e_{i}^{*}\right) \\
& =N^{*}(1+t)\left(\begin{array}{l}
n-1 \\
r-1
\end{array}\right)+t\left(\begin{array}{l}
n \\
r
\end{array}\right)(N-r h) \\
& =t\left(\left(\begin{array}{c}
n-1 \\
r-1
\end{array}\right) N^{*}+\left(\begin{array}{c}
n \\
r
\end{array}\right)(N-r h)\right)+N^{*}\left(\begin{array}{c}
n-1 \\
r-1
\end{array}\right) \\
& =t\left(\left(\begin{array}{c}
n-1 \\
r-1
\end{array}\right) N^{*}+\left(\begin{array}{c}
n-1 \\
r-1
\end{array}\right)(N-r h)+\left(\begin{array}{c}
n-1 \\
r
\end{array}\right)(N-r h)\right)+N^{*}\left(\begin{array}{c}
n-1 \\
r-1
\end{array}\right) \\
& =t N\left(\begin{array}{c}
n-1 \\
r
\end{array}\right)+N^{*}\left(\begin{array}{c}
n-1 \\
r-1
\end{array}\right) .
\end{aligned}
$$

Here we used the fact that $N+N^{*}=n h$ (as $W$ is a duality group) and $\left(\begin{array}{c}n-1 \\ r-1\end{array}\right)(n-r)=\left(\begin{array}{c}n-1 \\ r\end{array}\right) r$.

Independence over the fraction field. We now explain why the above Opdam-Gutkin calculation implies that Conjecture 4.1 may be shown with an independence argument.

Lemma 6.4. 28, Lemma 4.1] Let $A$ be a graded $k$-algebra and integral domain, and $M \cong A^{p}$ a free graded A-module whose homogeneous basis elements have degrees suming to $\psi$. Then another set of homogeneous elements $\left\{n_{1}, \cdots, n_{p}\right\}$ in $M$ with the same degree sum $\sum_{i=1}^{p} \operatorname{deg}\left(n_{i}\right)=\psi$ form an $A$-basis for $M$ if and only if they are linearly independent over the fraction field $K=\operatorname{Frac}(A)$.

Thus one may verify Conjecture 4.1 by proving that the basis elements of the module $\left(S\left(V^{*}\right) \otimes \wedge^{k} V^{*} \otimes\right.$ $\left.\wedge^{r} V\right)^{W}$ it predicts are linearly independent over the fraction field of $\left(S\left(V^{*}\right)\right.$ for each $k, r$ :

Proposition 6.5. Let $W$ be a coincidental reflection group with any set of basic invariants $f_{1}, \ldots, f_{n}$ and basic derivations $\theta_{1}, \ldots, \theta_{n}$. Fix some $1 \leq r \leq n$. If the derviation differential forms

$$
\tilde{\theta}_{i_{1}} \cdots \tilde{\theta}_{i_{k}}\left(\theta_{j_{1}} \wedge \cdots \wedge \theta_{j_{r}}\right) \in S\left(V^{*}\right) \otimes \wedge^{k} V^{*} \otimes \wedge^{r} V
$$

for $0 \leq k \leq r$ and $1 \leq i_{1}<\cdots<i_{k} \leq r$ and $1 \leq j_{1}<\cdots<j_{r} \leq n$ are linearly independent over the fraction field Frac $\left(S\left(V^{*}\right)\right)$, then they form a basis for $M_{r}=\left(S\left(V^{*}\right) \otimes \wedge V^{*} \otimes \wedge^{r} V\right)^{W}$ over the exterior subalgebra $R_{r}:=\wedge_{S\left(V^{*}\right)^{W}}\left\{d f_{1}, \ldots, d f_{n-r}\right\}$. In this case, $M_{r}$ is a free module over $R_{r}$.

Proof. Use Proposition 6.3 together with Lemma 6.4 and Lemma 5.1 .

By Proposition 6.5, it suffices to check various determinants are nonzero to prove Conjecture 4.1

\section{The Main Result And Molchanov'S hypothesis}

In this section, we outline the proof of our main result, Theorem 1.1 from the Introduction:

Theorem 1.1. For any coincidental complex reflection group $W$ acting on $V=\mathbb{C}^{n}$,

$$
\operatorname{Hilb}\left(\left(S\left(V^{*}\right) \otimes \wedge V^{*} \otimes \wedge V\right)^{W}, q, t, s\right)=\sum_{r=0}^{n} \sigma_{r}\left(q^{e_{1}^{*}}, \ldots, q^{e_{n}^{*}}\right) \frac{\prod_{i=1}^{r}\left(1+q^{e_{i}^{*}} t\right) \prod_{i=1}^{n-r}\left(1+q^{e_{i}} t\right) s^{r}}{\prod_{i=1}^{n}\left(1-q^{d_{i}}\right)} .
$$

Proof. We proceed in essentially three cases, some of which prove the stronger Conjecture 4.1; see Lemma4.4

- For the Weyl groups of type $A_{n}$ and the Shephard-Todd family $G(d, 1, n)$, Theorem 1.1 was deduced in Section 3. as Corollaries 3.4 and 3.16

- We prove Conjecture 4.1 uniformly in Section 8 for all coincidental groups of rank 2 , relying on Proposition 6.5 so as to only check that certain determinants are nonzero; see Theorem 8.4

- This leaves the exceptional real type $H_{3}$ and Shephard groups $G_{25}, G_{26}, G_{32}$ of ranks 3 or 4 , where we checked Conjecture 4.1 in Mathematica via Proposition 6.5 for these choices of $\left\{f_{i}\right\},\left\{\theta_{i}\right\}$ :

- For $H_{3}$, we used $\left\{f_{i}\right\}$ from Saito, Yano, and Sekiguchi [30], with $\theta_{i}=\sum_{j=1}^{n} \frac{\partial f_{i}}{\partial x_{j}} \otimes y_{j}$.

- For $G_{25}, G_{26}, G_{32}$, we used $\left\{f_{i}\right\}$ and $\left\{\theta_{i}\right\}$ from Orlik and Terao [26, Appendix B.3]. 
Remark 7.1. Theorem 1.1 is very closely related to a hypothesis stated by Molchanov [24, §8]. Molchanov's formulation 1 differs from Theorem 1.1 both in that he assumes that $W$ is a real reflection group (so that as $W$ representations, $V^{*} \cong V$ and $e_{i}^{*}=e_{i}$ ) and in that he assumes that the exponents are distinct. Unfortunately, the scope of his hypothesis is off, and Theorem 1.1 seems to be the correct formulation. In fact, combining Remark 3.19 with the data on the noncoincidental exceptional reflection groups presented in Section 11, one sees that the formula in Theorem 1.1 holds for an irreducible reflection group $W$, real or complex, if and only $W$ is coincidental.

\section{ReFLECTION GRoups In TWO Dimensions}

Here we verify Conjecture 4.1 for rank 2 coincidental reflection groups using Proposition 6.5. In two dimensions, the coincidental groups $W$ are the same as the irreducible duality groups. Recall as notaion that $W$ acts on $V=\mathbb{C}^{2}$ with $\mathbb{C}$-basis $\left\{y_{1}, y_{2}\right\}$ and on $V^{*}$ with dual $\mathbb{C}$-basis $\left\{x_{1}, x_{2}\right\}$, so that $S\left(V^{*}\right)=\mathbb{C}\left[x_{1}, x_{2}\right]$.

Lemma 8.1. For $W$ any rank 2 irreducible finite reflection group acting on $V=\mathbb{C}^{2}$, the $W$-invariant space $\left(V^{*} \otimes V^{*} \otimes \wedge^{2} V\right)^{W}$ is 1-dimensional over $\mathbb{C}$, spanned by

$$
\left(x_{1} \otimes x_{2}-x_{2} \otimes x_{1}\right) \otimes y_{1} \wedge y_{2} .
$$

Proof. Again, we write $S=S\left(V^{*}\right)$. As a GL $(V)$-representation, $V^{*} \otimes V^{*} \cong S^{2} \oplus \wedge^{2} V^{*}$. This isomorphism restricts one of $W$-representations, and hence

$$
\left(V^{*} \otimes V^{*} \otimes \wedge^{2} V\right)^{W} \cong\left(S^{2} \otimes \wedge^{2} V\right)^{W} \oplus\left(\wedge^{2} V^{*} \otimes \wedge^{2} V\right)^{W}
$$

We analyze the two direct summands in this last expression. Since $\wedge^{2} V$ is 1-dimensional spanned by $d y_{1} \wedge d y_{2}$ and carries the $W$-character det, for any $W$-representation $U$, the $W$-fixed space $\left(U \otimes \wedge^{2} V\right)^{W}$ will be the tensor product of the $\operatorname{det}^{-1}$-isotypic component of $U$ with $\mathbb{C} d y_{1} \wedge d y_{2}$.

For $U=\wedge^{2} V^{*}$, this det ${ }^{-1}$-isotypic component is 1-dimensional, spanned by $x_{1} \otimes x_{2}-x_{2} \otimes x_{1}$.

For $U=S^{2}$, we argue that this $\operatorname{det}^{-1}$-isotypic component will vanish. For reflection groups $W$ of any rank, the det ${ }^{-1}$-isotypic component of $S$ is the free $S^{W}$-module $S^{W} \cdot Q$ of rank 1 (by Stanley [38, Thm. 3.1], see also [26, Ex. 6.40]) for $Q$ the product of the linear forms defining the reflecting hyperplanes with degree $N^{*}=e_{1}^{*}+\cdots+e_{n}^{*}$, the number of reflecting hyperplanes. But $N^{*}>2$ for any irreducible reflection group $W$ of rank at least 2 and hence $S^{2}$ has zero intersection with this $\operatorname{det}^{-1}$-isotypic component.

Thus $\left(V^{*} \otimes V^{*} \otimes \wedge^{2} V\right)^{W} \cong\left(\wedge^{2} V^{*} \otimes \wedge^{2} V\right)^{W}$ is spanned by $\left(x_{1} \otimes x_{2}-x_{2} \otimes x_{1}\right) \otimes y_{1} \wedge y_{2}$.

Proposition 8.3. If $h\left(x_{1}, \ldots, x_{n}\right)$ in $S\left(V^{*}\right)$ is homogeneous and nonzero, then $\bar{h}\left(\frac{\partial}{\partial x_{1}}, \ldots, \frac{\partial}{\partial x_{n}}\right)(h) \neq 0$.

Proof. Express $h$ of degree $d$ as a finite sum of monomials,

$$
h=\sum_{\substack{\alpha=\left(\alpha_{1}, \ldots, \alpha_{n}\right): \\ \sum_{i} \alpha_{i}=d}} c_{\alpha} x_{1}^{\alpha_{1}} \cdots x_{n}^{\alpha_{n}}=\sum_{\alpha} c_{\alpha} \mathbf{x}^{\alpha},
$$

and write $\left(\partial x_{i}\right)^{m}$ for $\partial^{m} / \partial x_{i}^{m}$. For $\sum_{i} \alpha_{i}=\sum_{i} \beta_{i}$, the monomial $\left(\partial^{\alpha_{1}}\right)^{\alpha_{1}} \cdots\left(\partial x_{n}\right)^{\alpha_{n}}\left(\mathbf{x}^{\beta}\right)$ is $\alpha_{1} ! \cdots \alpha_{n} !$ if $\beta=\alpha$ but vanishes for $\beta \neq \alpha$. Thus by linearity,

$$
\bar{h}\left(\partial x_{1}, \ldots, \partial x_{n}\right)(h)=\sum_{\alpha, \beta} \bar{c}_{\alpha} c_{\alpha}\left(\partial x_{1}\right)^{\alpha_{1}} \cdots\left(\partial x_{n}\right)^{\alpha_{n}}\left(\mathbf{x}^{\beta}\right)=\sum_{\alpha}|c|^{2} \alpha_{1} ! \cdots \alpha_{n} !,
$$

which is strictly positive as long as at least one $c_{\alpha} \neq 0$ in $\mathbb{C}$, that is, as long as $h \neq 0$ in $S$.

Proposition 8.4. Conjecture 4.1 holds for any irreducible duality group $W$ acting on $\mathbb{C}^{2}$ : For $S=S\left(V^{*}\right)$ and $M_{r}=\left(S \otimes \wedge V^{*} \otimes \wedge^{r} V\right)^{W}$, there is a set of basic invariants $f_{1}, f_{2}$ and basic derivations $\theta_{1}, \theta_{2}$ so that

- $M_{0}$ is free over $R_{0}=\wedge_{S W}\left\{d f_{1}, d f_{2}\right\}$ with basis $\{1\}$;

- $M_{1}$ is free over $R_{1}=S^{W} d f_{1}$ with basis $\left\{\theta_{1}, \theta_{2}, \tilde{\theta}_{1}\left(\theta_{1}\right), \tilde{\theta}_{1}\left(\theta_{2}\right)\right\}$;

- $M_{2}$ is free over $R_{2}=S^{W}$ with basis $\left\{\theta_{1} \wedge \theta_{2}, \tilde{\theta}_{1}\left(\theta_{1} \wedge \theta_{2}\right), \tilde{\theta}_{2}\left(\theta_{1} \wedge \theta_{2}\right), \tilde{\theta}_{1} \tilde{\theta}_{2}\left(\theta_{1} \wedge \theta_{2}\right)\right\}$.

\footnotetext{
${ }^{1}$ Warning: translation of his paper to English introduced two typos-instead of $\prod_{i=1}^{r}$ and $\prod_{i=1}^{n}$, it has $\prod_{i=1}^{m}$ for both.
} 
Proof. Recall from Remark 4.3 that Conjecture 4.1 is known for all duality groups when $r \in\{0,1\}$ or $k=0$. Since $0 \leq r \leq n=2$, it hence suffices to only consider here the case $r=n=2$ and $k \in\{1,2\}$.

By Proposition 6.5. we need only check the linear independence over the fraction field $K$ of $S$ of

$$
\tilde{\theta}_{1}\left(\theta_{1} \wedge \theta_{2}\right) \text { and } \tilde{\theta}_{2}\left(\theta_{1} \wedge \theta_{2}\right) \quad \text { in } \quad M_{2,1}:=\left(S \otimes \wedge^{1} V^{*} \otimes \wedge^{2} V\right)^{W} \quad(\text { the case } k=1)
$$

and also check that

$$
0 \neq \tilde{\theta}_{1} \tilde{\theta}_{2}\left(\theta_{1} \wedge \theta_{2}\right) \quad \text { in } \quad M_{2,2}:=\left(S \otimes \wedge^{2} V^{*} \otimes \wedge^{2} V\right)^{W} \quad(\text { the case } k=2) .
$$

Recall that $\theta_{1} \wedge \theta_{2}=Q \otimes 1 \otimes y_{1} \wedge y_{2}$ by [25, Thm. 3.1] as $\wedge^{2} V=\mathbb{C} y_{1} \wedge y_{2}$. Again, we write $\partial x_{i}$ for $\partial / \partial x_{i}$.

The case $n=r=2$ and $k=2$. The derivation differential form

$$
\tilde{\theta}_{1} \tilde{\theta}_{2}\left(\theta_{1} \wedge \theta_{2}\right)=\tilde{\theta}_{1} \tilde{\theta}_{2}\left(Q \otimes 1 \otimes y_{1} \wedge y_{2}\right)=\bar{Q}\left(\partial x_{1}, \partial x_{2}\right)(Q) \otimes x_{1} \wedge x_{2} \otimes y_{1} \wedge y_{2}
$$

is nonzero since Lemma 8.3 implies that the scalar $\bar{Q}\left(\partial x_{1}, \partial x_{2}\right)(Q)$ in $\mathbb{C}$ is nonzero.

The case $n=r=2$ and $k=1$. Here, one must check that

$$
\begin{aligned}
& \omega_{1}:=\tilde{\theta}_{1}\left(\theta_{1} \wedge \theta_{2}\right)=\tilde{\theta}_{1}\left(Q \otimes 1 \otimes y_{1} \wedge y_{2}\right) \quad \text { and } \\
& \omega_{2}:=\tilde{\theta}_{2}\left(\theta_{1} \wedge \theta_{2}\right)=\tilde{\theta}_{2}\left(Q \otimes 1 \otimes y_{1} \wedge y_{2}\right)
\end{aligned}
$$

are $K$-linearly independent in $S \otimes V^{*} \otimes \wedge^{2} V$. Our strategy is to make convenient choices for basic derivations $\theta_{1}, \theta_{2}$ that identify $\omega_{1}, \omega_{2}$ more concretely. As explained in [26, Appendix B.2], one may choose in rank 2

$$
\begin{aligned}
& \theta_{1}=x_{1} \otimes 1 \otimes y_{1} \quad+x_{2} \otimes 1 \otimes y_{2} \quad \text { and } \\
& \theta_{2}=-\frac{\partial Q}{\partial x_{2}} \otimes 1 \otimes y_{1} \quad+\quad \frac{\partial Q}{\partial x_{1}} \otimes 1 \otimes y_{2} .
\end{aligned}
$$

With this choice,

$$
\begin{aligned}
& \omega_{1}=\left(\frac{\partial Q}{\partial x_{1}} \otimes x_{1}+\frac{\partial Q}{\partial x_{2}} \otimes x_{2}\right) \otimes y_{1} \wedge y_{2} \quad \text { and } \\
& \omega_{2}=\left(-\frac{\overline{\partial Q}}{\partial x_{2}}\left(\partial x_{1}, \partial x_{2}\right)(Q) \otimes x_{1}+\overline{\frac{\partial Q}{\partial x_{1}}}\left(\partial x_{1}, \partial x_{2}\right)(Q) \otimes x_{2}\right) \otimes y_{1} \wedge y_{2} .
\end{aligned}
$$

Note that $\omega_{2}$ has $S$-degree 1 inside $S \otimes V^{*} \otimes \wedge^{2} V^{*}$. Also notice that $\omega_{2} \neq 0$, since otherwise

$$
\overline{\frac{\partial Q}{\partial x_{1}}}\left(\partial x_{1}, \partial x_{2}\right)(Q)=\overline{\frac{\partial Q}{\partial x_{2}}}\left(\partial x_{1}, \partial x_{2}\right)(Q)=0
$$

which would then imply the following contradiction to Proposition 8.3

$$
\begin{aligned}
0=\frac{\partial}{\partial x_{1}}\left(\overline{\frac{\partial Q}{\partial x_{1}}}\left(\partial x_{1}, \partial x_{2}\right)(Q)\right)+\frac{\partial}{\partial x_{2}}\left(\overline{\frac{\partial Q}{\partial x_{2}}}\left(\partial x_{1}, \partial x_{2}\right)(Q)\right) & =\left(\overline{x_{1} \frac{\partial Q}{\partial x_{1}}}+\overline{x_{2} \frac{\partial Q}{\partial x_{2}}}\right)\left(\partial x_{1}, \partial x_{2}\right)(Q) \\
& =\operatorname{deg}(Q) \cdot \bar{Q}\left(\partial x_{1}, \partial x_{2}\right)(Q) .
\end{aligned}
$$

Thus $\omega_{2}$ is a nonzero element of $\left(V^{*} \otimes V^{*} \otimes \wedge^{2} V\right)^{W}$ by Lemma 5.1 and we may identify $\omega_{2}$, up to a nonzero scalar, with $\left(x_{1} \otimes x_{2}-x_{2} \otimes x_{1}\right) \otimes y_{1} \wedge y_{2}$ by Lemma 8.1 .

Finally, we check that $\omega_{1}, \omega_{2}$ are $K$-linearly independent. The matrix expressing $\omega_{1}, \omega_{2}$ with respect to the $S$-basis $x_{1} \otimes y_{1} \wedge y_{2}, x_{2} \otimes y_{1} \wedge y_{2}$ of $S \otimes V^{*} \otimes \wedge^{2} V$ has determinant $x_{1} \frac{\partial Q}{\partial x_{1}}+x_{2} \frac{\partial Q}{\partial x_{2}}=\operatorname{deg}(Q) \cdot Q \neq 0$.

\section{Conversion Between $(q, t)$-Analogues of $h$-vectors and $f$-vectors}

We now highlight some applications of Theorem 1.1 to $f$-vectors and $h$-vectors in algebraic combinatorics. We will see in Remark 10.6 later how these applications answer a question on $q$-Kirkman and $q$-Narayana numbers raised in [2, Problem 11.3].

Let $W$ be a coincidental reflection group with smallest exponent $e_{1}$ and exponent gap $a$. In the Introduction, we defined these $(q, t)$-analogues of the $f$-vector and $h$-vector:

$$
\begin{aligned}
& \left.f_{r}(W ; q, t)=q^{r+a\left(\begin{array}{c}
r \\
2
\end{array}\right)}\left[\begin{array}{l}
n \\
r
\end{array}\right]_{q^{a}} \frac{\left(-t q^{e_{1}} ; q^{a}\right)_{n-r}\left(-t q^{-1} ; q^{-a}\right)_{r}}{\left(q^{e_{1}+1} ; q^{a}\right)_{n}} \quad \text { (the }(q, t) \text {-analogue of the } f \text {-vector }\right), \\
& \left.h_{r}(W ; q, t)=\left(-t q^{-a r-1}\right)^{n-r}\left[\begin{array}{l}
n \\
r
\end{array}\right]_{q^{a}} \frac{\left(-t q^{-1} ; q^{-a}\right)_{r}}{\left(q^{e_{1}+1} ; q^{a}\right)_{r}} \quad \text { (the }(q, t) \text {-analogue of the } h \text {-vector }\right) .
\end{aligned}
$$


We later explain in Section 10 why it is appropriate to call these analogues of $f$-vectors and $h$-vectors (see (10.7) and (10.8)). In this section, we prove Theorem 1.5 of the Introduction that converts between these $(q, t)$-analogues. As preparation, we rephrase $f_{r}(W ; q, t)$ using the notation of basic hypergeometric functions [9, Chap. 1]:

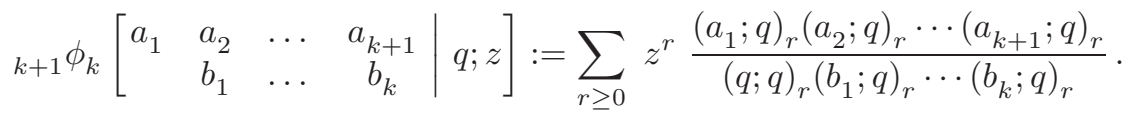

Proposition 9.3. The following two statements are equivalent reformulations of Theorem [.1;

(a) $f_{r}(W ; q, t)=\frac{1}{|W|} \sum_{w \in W} \frac{\operatorname{det}(1+t w)}{\operatorname{det}(1-q w)}=t^{r}\left[\begin{array}{l}n \\ r\end{array}\right]_{q^{a}} \frac{\left(-t q^{e_{1}} ; q^{a}\right)_{n-r}\left(-q t^{-1} ; q^{a}\right)_{r}}{\left(q^{e_{1}+1} ; q^{a}\right)_{n}}$.

(b) $\sum_{r=0}^{n} s^{r} f_{r}(W ; q, t)=\frac{1}{|W|} \sum_{w \in W} \frac{\operatorname{det}\left(1+s w^{-1}\right) \operatorname{det}(1+t w)}{\operatorname{det}(1-q w)}$

$$
=\frac{\left(-t q^{e_{1}} ; q^{a}\right)_{n}}{\left(q^{e_{1}+1} ; q^{a}\right)_{n}}{ }_{2} \phi_{1}\left[\begin{array}{cc|c}
q^{-a n} & -q t^{-1} & q^{a} ;-s q^{a-e_{1}}
\end{array}\right] .
$$

Proof. For Equation (a), we simply rewrite Theorem 1.1 with

$$
f_{r}(W ; q, t)=q^{r+a\left(\begin{array}{c}
r \\
2
\end{array}\right)}\left[\begin{array}{c}
n \\
r
\end{array}\right]_{q^{a}} \frac{\left(-t q^{e_{1}} ; q^{a}\right)_{n-r}\left(-t q^{-1} ; q^{-a}\right)_{r}}{\left(q^{e_{1}+1} ; q^{a}\right)_{n}}=t^{r}\left[\begin{array}{l}
n \\
r
\end{array}\right]_{q^{a}} \frac{\left(-t q^{e_{1}} ; q^{a}\right)_{n-r}\left(-q t^{-1} ; q^{a}\right)_{r}}{\left(q^{e_{1}+1} ; q^{a}\right)_{n}}
$$

by applying this easy fact (see $\left[9\right.$, eqn. (I.3)]) to the numerator factor $\left(-t q^{-1} ; q^{-a}\right)_{r}$ :

$$
\left(z ; q^{-1}\right)_{r}=z^{r} q^{-\left(\begin{array}{c}
r \\
2
\end{array}\right)}\left(-z^{-1} ; q\right)_{r} .
$$

To see (b), we reexpress (a) as

$$
f_{r}(W ; q, t)=t^{r} \frac{\left(q^{a} ; q^{a}\right)_{n}}{\left(q^{e_{1}+1} ; q^{a}\right)_{n}} \frac{\left(-t q^{e_{1}} ; q^{a}\right)_{n-r}}{\left(q^{a} ; q^{a}\right)_{n-r}} \frac{\left(-q t^{-1} ; q^{a}\right)_{r}}{\left(q^{a} ; q^{a}\right)_{r}} .
$$

We rewrite the middle quotient in the product by applying the identity (see [9. (I.11)])

$$
\frac{(w ; q)_{n-r}}{(z ; q)_{n-r}}=\frac{(w ; q)_{n}}{(z ; q)_{n}} \frac{\left(q^{1-n} / z ; q\right)_{r}}{\left(q^{1-n} / w ; q\right)_{r}}\left(\frac{z}{w}\right)^{r}
$$

with $w=-t q^{e_{1}}, z=q^{a}$ and obtain

$$
f_{r}(W ; q, t)=\frac{\left(-t q^{e_{1}} ; q^{a}\right)_{n}}{\left(q^{e_{1}+1} ; q^{a}\right)_{n}}\left(-q^{a-e_{1}}\right)^{r} \frac{\left(q^{-a n} ; q^{a}\right)_{r}}{\left(q^{a} ; q^{a}\right)_{r}} \frac{\left(-q t^{-1} ; q^{a}\right)_{r}}{\left(-q^{a(1-n)-e_{1}} t^{-1} ; q^{a}\right)_{r}},
$$

which then immediately gives (b) from definition (9.2) of ${ }_{2} \phi_{1}$.

We can now extend the relation between $f$ - and $h$-vectors for $n$-dimensional simple polytopes, namely,

$$
\sum_{r=0}^{n} s^{r} f_{r}=\sum_{r=0}^{n}(1+s)^{r} h_{r}
$$

to a relation between the above $(q, t)$-analogues; we prove the theorem from the Introduction:

Theorem 1.5. For any coincidental reflection group $W$ with exponent gap a,

$$
\sum_{r=0}^{n} s^{r} f_{r}(W ; q, t)=\sum_{r=0}^{n}\left(-s q ; q^{a}\right)_{r} \cdot h_{r}(W ; q, t) .
$$

Proof. We apply a terminating form of Jackson's ${ }_{2} \phi_{1}$-transformation 2] [9, III.7],

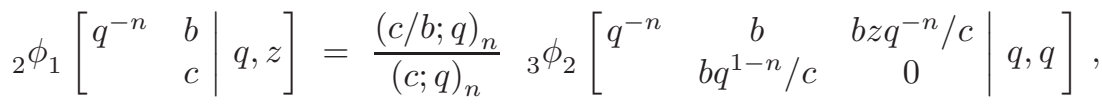

\footnotetext{
${ }^{2}$ The authors thank Dennis Stanton for pointing them to this identity.
} 
to the ${ }_{2} \phi_{1}$-expression in Proposition 9.3(b) after first replacing $q$ by $q^{a}$ and then selecting parameter choices

$$
b=-q t^{-1}, \quad c=-q^{a(1-n)-e_{1}} t^{-1}, \quad z=-s q^{a-e_{1}} .
$$

Since

$$
c / b=q^{a(1-n)-e_{1}-1}, \quad b q^{1-n} / c=q^{e_{1}+1}, \quad b z q^{-1} / c=-s q,
$$

and $(z ; q)_{n}=\left(q^{1-n} / z ; q\right)_{n}(-z)^{n} q^{\left(\begin{array}{c}n \\ 2\end{array}\right)}$ (see [9, eqn. (I.7)]), one can rewrite the quotient in (9.6) as

$$
\frac{(c / b ; q)_{n}}{(c ; q)_{n}}=\frac{\left(q^{a(1-n)-e_{1}-1} ; a^{a}\right)_{n}}{\left(-t^{-1} q^{a(1-n)-e_{1}} ; q^{a}\right)_{n}}=\frac{\left(q^{e_{1}+1} ; q^{a}\right)_{n}}{\left(-t q^{e_{1}} ; q^{a}\right)_{n}}\left(-t q^{-1}\right)^{n} .
$$

Thus Proposition 9.3(b) and (9.6) imply that

$$
\begin{aligned}
& \sum_{r=0}^{n} s^{r} f_{r}(W ; q, t)=\left(-t q^{-1}\right)^{n} \cdot{ }_{3} \phi_{2}\left[\begin{array}{ccc|c}
q^{-a n} & -q t^{-1} & -s q & q^{a}, q^{a} \\
& q^{e_{1}+1} & 0
\end{array}\right] \\
& =\left(-t q^{-1}\right)^{n} \sum_{r=0}^{n}\left(-s q ; q^{a}\right)_{r} \cdot q^{a r} \frac{\left(q^{-a n} ; q^{a}\right)_{r}\left(-q t^{-1} ; q^{a}\right)_{r}}{\left(q^{a} ; q^{a}\right)_{r}\left(q^{e_{1}+1} ; q^{a}\right)_{r}} .
\end{aligned}
$$

It remains to check that

$$
\left(-t q^{-a r-1}\right)^{n-r}\left[\begin{array}{l}
n \\
r
\end{array}\right]_{q^{a}} \frac{\left(-t q^{-1} ; q^{-a}\right)_{r}}{\left(q^{e_{1}+1} ; q^{a}\right)_{r}}=\left(-t q^{-1}\right)^{n} q^{a r} \frac{\left(q^{-a n} ; q^{a}\right)_{r}\left(-q t^{-1} ; q^{a}\right)_{r}}{\left(q^{a} ; q^{a}\right)_{r}\left(q^{e_{1}+1} ; q^{a}\right)_{r}} .
$$

We substitute definition (1.2) of the $q$-binomial and cancel common factors to see that (9.7) is equivalent to

$$
\left(-t q^{-a r-1}\right)^{n-r} \frac{\left(q^{a} ; q^{a}\right)_{n}}{\left(q^{a} ; q^{a}\right)_{n-r}}\left(-t q^{-1} ; q^{-a}\right)_{r}=\left(-t q^{-1}\right)^{n} q^{a r}\left(q^{-a n} ; q^{a}\right)_{r}\left(-q t^{-1} ; q^{a}\right)_{r} .
$$

We verify this last equality by applying (9.4) to the factor $\left(-t q^{-1} ; q^{-a}\right)_{r}$ on the left and rewriting the factor $\left(q^{-a n} ; q^{a}\right)_{r}$ on the right using this fact (from [9, I.12]) with $q$ replaced by $q^{a}$ :

$$
\left(q^{-n} ; q\right)_{r}=\frac{(q ; q)_{n}}{(q ; q)_{n-r}}(-1)^{r} q^{\left(\begin{array}{c}
r \\
2
\end{array}\right)-n r}
$$

\section{The connection with Catalan, Kirkman, Narayana, Cambrian and Clusters}

We explain here how specializations of our product formulas for $f_{r}(W ; q, t)$ and $h_{r}(W ; q, t)$ give known product formulas for $q$-Catalan, $q$-Kirkman, and $q$-Narayana numbers. We also see how their $q=1$ specializations give the $f$-vectors and $h$-vectors for Cambrian and cluster fans. This starts with certain graded representations of a reflection group $W$ called graded parking spaces.

The graded parking spaces. For a positive integer $p$, define a class function $\chi^{(p)}: W \longrightarrow \mathbb{Q}(q)$ by

$$
\chi^{(p)}(w):=\frac{\operatorname{det}\left(1-q^{p} w\right)}{\operatorname{det}(1-q w)} .
$$

For special values of $p$, the function $\chi^{(p)}$ is actually a $\mathbb{Z}[q]$-valued class function and even turns out to be the graded character of a genuine $W$-representation. Ito and Okada 17 tabulated the values of $p$ for which this holds for each irreducible complex reflection group. For duality groups $W$, these special values of $p$ include all the Fuss-Catalan cases, that is, cases where $p \equiv 1 \bmod h$ for $h=d_{n}=\max \left\{d_{i}\right\}$ :

- This fact is related to work of Haiman [12, $\S 7$ for Weyl groups, where $\chi^{(p)}$ gives a graded version of the $p$-parking space $W$-permutation representation on $R / p R$, in which $R$ is the root lattice for $W$.

- It holds more generally for real reflection groups $W$ via results from representation theory of rational Cherednik algebras, e.g., see [3, Remark 4.4]; Gordon and Griffeth [10, §1.6] generalize these ideas to all complex reflection groups.

- One may verify this fact from Ito and Okada's tabulation [17, Table 1], where it only fails for the four non-duality groups $G_{12}, G_{13}, G_{22}$, and $G_{31}$. 
The $q$-Kirkman numbers. In the cases where $\chi^{(p)}$ is a genuine graded character, setting $t=-q^{p}$ in Proposition 9.3 (a) gives an expression for the (graded) multiplicity of the $W$-irreducibles $\wedge^{r} V$ in $\chi^{(p)}$ :

$$
f_{r}\left(W ; q,-q^{p}\right)=\left\langle\chi^{(p)}, \wedge^{r}(V)\right\rangle_{W}=(-1)^{r} q^{p r}\left[\begin{array}{l}
n \\
r
\end{array}\right]_{q^{a}} \frac{\left(q^{p+e_{1}} ; q^{a}\right)_{n-r}\left(q^{p-1} ; q^{-a}\right)_{r}}{\left(q^{e_{1}+1} ; q^{a}\right)_{n}} .
$$

For real reflection groups $W$, these graded multiplicities are called $q$-Kirkman numbers; see [2, $\S 9, \S 11]$. Specializing (10.1) to the case of types $A$ (where $e_{1}=1=a$ ) and $B / C$ (where $e_{1}=1, a=2$ ) gives

$$
\begin{aligned}
f_{r}\left(A_{n-1} ; q,-q^{p}\right) & =q^{\left(\begin{array}{c}
r+1 \\
2
\end{array}\right)} \frac{1}{[p]_{q}}\left[\begin{array}{cl}
n-1 \\
r
\end{array}\right]_{q}\left[\begin{array}{c}
p+n-r-1 \\
n
\end{array}\right]_{q} & \text { for } \operatorname{gcd}(n, p)=1 \text { and } \\
f_{r}\left(B_{n} / C_{n} ; q,-q^{p}\right) & =q^{r^{2}}\left[\begin{array}{c}
\frac{p-1}{2} \\
r
\end{array}\right]_{q^{2}}\left[\begin{array}{c}
\frac{p-1}{2}+n-r \\
n-r
\end{array}\right]_{q} & \text { for } p \text { odd. }
\end{aligned}
$$

The special case $p=h+1$ was listed in 2. In verifying that (10.1) specializes to these two formulas, it is helpful to note that $p \equiv 1 \bmod a$ in these cases, and so one can again use (9.8) to rewrite (10.1) as

$$
f_{r}\left(W ; q,-q^{p}\right)=q^{r+a\left(\begin{array}{c}
r \\
2
\end{array}\right)}\left[\begin{array}{l}
n \\
r
\end{array}\right]_{q^{a}} \frac{\left(q^{p+e_{1}} ; q^{a}\right)_{n-r}\left(q^{a} ; q^{a}\right)_{\frac{p-1}{a}}}{\left(q^{e_{1}+1} ; q^{a}\right)_{n}\left(q^{a} ; q^{a}\right)_{\frac{p-1}{a}-r}} .
$$

The $q$-Catalans and $q$-Narayanas. Setting $s=0$ in Theorem 1.5 gives the identity

$$
\frac{\left(-t q^{e_{1}} ; q^{a}\right)}{\left(q^{e_{1}+1} ; q^{a}\right)_{n}}=f_{0}(W ; q, t)=\sum_{r=0}^{n} h_{r}(W ; q, t) .
$$

In the cases where $\chi^{(p)}$ is a genuine graded character, setting $t=-q^{p}$ in the left side of (10.2) gives what one might call the $p$-rational version of the $q$-Catalan number for $W$ :

$$
\frac{\left(q^{p+e_{1}} ; q^{a}\right)}{\left(q^{1+e_{1}} ; q^{a}\right)_{n}}=\prod_{i=1}^{n} \frac{1-q^{p+e_{i}}}{1-q^{1+e_{i}}}=: \operatorname{Cat}^{(p)}(W, q) .
$$

Here, the first equality assumes $W$ is coincidental with smallest exponent $e_{1}$ and exponent gap $q$. When $p=h+1$, this product is called the $q$-Catalan number for $W$; see, e.g. Armstrong [1]. Thus setting $t=-q^{p}$ in (10.2) gives a summation formula for coincidental reflection groups $W$ :

$$
\operatorname{Cat}^{(p)}(W, q)=\frac{\left(q^{p+e_{1}} ; q^{a}\right)}{\left(q^{e_{1}+1} ; q^{a}\right)_{n}}=\sum_{r=0}^{n} h_{r}\left(W ; q,-q^{p}\right)
$$

where each summand has the explicit product formula

$$
h_{r}\left(W ; q,-q^{p}\right)=\left(q^{p-a r-1}\right)^{n-r}\left[\begin{array}{l}
n \\
r
\end{array}\right]_{q^{a}} \frac{\left(q^{p-1} ; q^{-a}\right)_{r}}{\left(q^{e_{1}+1} ; q^{a}\right)_{r}} .
$$

Happily, (10.3) agrees with the type $A$ and $B / C$ formulas for the $q$-Narayana numbers computed in [29]:

$$
\begin{aligned}
h_{r}\left(A_{n-1} ; q,-q^{p}\right) & =q^{(n-1-r)(p-1-r)} \frac{1}{[r+1]_{q}}\left[\begin{array}{cl}
n-1 \\
r
\end{array}\right]_{q}\left[\begin{array}{c}
p-1 \\
r
\end{array}\right]_{q} & \text { for } \operatorname{gcd}(n, p)=1, \\
h_{r}\left(B_{n} / C_{n} ; q,-q^{p}\right) & =\left(q^{2}\right)^{(n-r)\left(\frac{p-1}{2}-r\right)}\left[\begin{array}{c}
n \\
r
\end{array}\right]_{q^{2}}\left[\begin{array}{c}
\frac{p-1}{2} \\
r
\end{array}\right]_{q^{2}} & \text { for } p \text { odd. }
\end{aligned}
$$

In verifying this, it is helpful to again use (9.4) to replace $\left(q^{p-1} ; q^{-a}\right)_{r}$ in (10.3) with a multiple of $\left(q^{1-p} ; q^{a}\right)_{r}$ and then to further use that $p \equiv 1 \bmod a$ in these cases and employ (9.8) again, rewriting (10.3) as

$$
h_{r}\left(W ; q,-q^{p}\right)=q^{(n-r)(p-a r-1)}\left[\begin{array}{l}
n \\
r
\end{array}\right]_{q^{a}} \frac{\left(q^{a} ; q^{a}\right)_{\frac{p-1}{a}}}{\left(q^{e_{1}+1} ; q^{a}\right)_{n}\left(q^{a} ; q^{a}\right)_{\frac{p-1}{a}-r}} .
$$

Remark 10.5. We say "happily" above because the formulas in (10.4) came from a subtle and general Weyl group construction that arose from work of the third author [36], described in [29, that always produces $q$-Narayana numbers summing to $q$-Catalan numbers. But there was nothing, a priori, indicating that they must coincide with the values $h_{r}\left(W ; q,-q^{p}\right)$ arising naturally here for each coincidental reflection group $W$. 
Remark 10.6. For the coincidental types, setting $t=-q^{p}$ in Theorem 1.5 relates the $q$-Kirkman numbers $f_{r}\left(W ; q,-q^{p}\right)$ to the $q$-Narayana numbers $h_{r}\left(W ; q,-q^{p}\right)$, as asked for in [2, Problem 11.3].

The $f$-vector and $h$-vector. Further specializing to $t=-q^{h+1}$ (so $p=h+1$ ) and taking $q \rightarrow 1$ in (9.1) produces integers for a real reflection group $W$

$$
\begin{aligned}
& f_{r}:=\left[f_{r}\left(W ; q,-q^{h+1}\right)\right]_{q=1} \text { and } \\
& h_{r}:=\left[h_{r}\left(W ; q,-q^{h+1}\right)\right]_{q=1}
\end{aligned}
$$

that were observed in $[2, \S 3.3]$ to be the $f$-vector and h-vector, respectively, for the (finite type) cluster complexes of Fomin and Zelevinsky (in the Weyl group case) and Cambrian fans of Reading (for arbitrary real reflection groups). Specifically, $f_{r}$ counts the number of clusters of cardinality $n-r$, or the number of cones in the fan having dimension $n-r$. Thus Theorem 1.5 specializes in this instance to the usual $h$ vector-to- $f$-vector relationship for the simplicial spheres associated to these fans, or to the simple polytopes for which they are the normal fans, constructed by Hohlwedg, Lange, and Thomas [16. This again answers the second part of the question raised in [2, Problem 11.3] for (real) coincidental groups $W$.

Remark 10.9. Theorem 1.1 explains a mysterious product formula observed by Fomin and Reading [7, Thm. 8.5 at $m=1$ ] for the number of $r$-dimensional cones in the cluster/Cambrian fan for real coincidental reflection groups $W$ :

$$
f_{r}=\left(\begin{array}{l}
n \\
r
\end{array}\right) \prod_{i=1}^{n-r} \frac{h+d_{i}}{d_{i}}
$$

The formula follows from computing (10.7) by setting $t=-q^{h+1}$ and then $q=1$ in Theorem 1.1

$$
f_{r}=\left[f_{r}\left(W ; q,-q^{h+1}\right)\right]_{q=1}=\left(\begin{array}{c}
n \\
r
\end{array}\right) \frac{\prod_{i=1}^{r}\left(h+1-e_{i}^{*}\right) \cdot \prod_{i=1}^{n-r}\left(h+1+e_{i}\right)}{\prod_{i=1}^{n} d_{i}}=\left(\begin{array}{c}
n \\
r
\end{array}\right) \prod_{i=1}^{n-r} \frac{h+d_{i}}{d_{i}}
$$

where the last equality uses the fact that $d_{i}=e_{i}+1$ and that $e_{i}^{*}=h-e_{n+1-i}$ for real reflection groups $W$.

\section{Data on the NON-COINCIDENTAL EXCEPTIONAL GROUPS}

For the non-coincidental exceptional irreducible reflection groups $W$, we tabulate here the polynomials

$$
\nu_{r}(W, q, t):=\frac{\operatorname{Hilb}\left(\left(S\left(V^{*}\right) \otimes \wedge V^{*} \otimes \wedge^{r} V\right)^{W}, q, t\right)}{\operatorname{Hilb}\left(S\left(V^{*}\right)^{W}, q\right)}
$$

for $r=0,1,2, \ldots, n$. The results of Section 3 including Corollary 3.4 3.16, 3.18, give the same data for the Weyl groups of type $A$ and the infinite family $G(d e, e, n)$ of complex reflection groups. Thus, together with Theorem 1.1, this completes those calculations for all irreducible complex reflection groups. Also, together with Remark 3.19, it allows one to check that the answers in the non-coincidental cases always differ from what Theorem 1.1 would have predicted.

In this tabulation, we may assume without loss of generality that $1 \leq r \leq n-1$, since

$$
\begin{aligned}
& \nu_{0}(W, q, t)=\prod_{i=1}^{n}\left(1+t q^{e_{i}}\right) \quad \text { and } \\
& \nu_{n}(W, q, t)=\prod_{i=1}^{n}\left(q^{e_{i}^{*}}+t\right)
\end{aligned}
$$

for all reflection groups by Solomon's Theorem [35] and Theorem 2.10, respectively. Additionally, for duality groups $W$, we may assume $2 \leq r \leq n-1$, since equation (2.9) (see [28, eqn. (2.1)]) implies that

$$
\nu_{1}(W, q, t)=\left(\sum_{i=1}^{n} q^{e_{i}}\right)\left(1+t q^{-1}\right) \prod_{i=1}^{n-1}\left(1+t q^{e_{i}}\right) .
$$

In general, we will use the notation $[m]_{q}:=1+q+q^{2}+\cdots+q^{m-1}$. 
Rank 2 groups. For rank 2 complex reflection groups, one has a formula for $\nu_{1}(W, q, t)$ from [28, Cor. 10.2]

$$
\nu_{1}(W, q, t)=\left(1+t q^{-1}\right)\left(\left(q^{e_{1}^{*}}+q^{e_{2}^{*}}\right)+t\left(q^{e_{1}+1}+q^{e_{2}+1}\right)\right) .
$$

It was noted there that this agrees with equation (11.3), and hence also with Theorem 1.1] exactly when $W$ is a rank 2 duality group, or equivalently, a rank 2 coincidental group.

Real but non-coincidental reflection groups of rank at least 3. For real reflection groups, we may assume that $2 \leq r \leq\left\lfloor\frac{n}{2}\right\rfloor$, as they are all duality groups and additionally satisfy (see [28, Prop. 13.1])

$$
\nu_{n-r}(W, q, t)=t^{n} \nu_{r}\left(W, q, t^{-1}\right) .
$$

\begin{tabular}{|c|c|}
\hline$F_{4}$ & exponents $(1,5,7,11)$ \\
\hline \hline$\nu_{2}$ & $(q+t)[2]_{q^{4}}(1+t q) \cdot$ \\
& $\left(\left(q^{5}+q^{7}-q^{9}+q^{11}+q^{13}\right)\left(1+t^{2}\right)+\left(1+q^{6}+q^{8}+q^{10}+q^{12}+q^{18}\right) t\right)$ \\
\hline
\end{tabular}

\begin{tabular}{|c|c|}
\hline$H_{4}$ & exponents $(1,11,19,29)$ \\
\hline \hline$\nu_{2}$ & $(q+t)(1+t q) \cdot$ \\
& $\left(\left(q^{11}+q^{19}+2 q^{29}+q^{39}+q^{47}\right)\left(1+t^{2}\right)\right.$ \\
& $\left.+\left(1+q^{10}+q^{18}+q^{20}+q^{22}+q^{28}+q^{30}+q^{36}+q^{38}+q^{40}+q^{48}+q^{58}\right) t\right)$ \\
\hline
\end{tabular}

\begin{tabular}{|c|c|}
\hline$E_{6}$ & exponents $(1,4,5,7,8,11)$ \\
\hline \hline$\nu_{2}$ & $(q+t)[3]_{q^{3}}\left(1+q+q^{4}+q^{7}+q^{8}\right) \prod_{i=1}^{3}\left(1+t q^{e_{i}}\right)$. \\
& $\left(q^{4}+t \frac{[2]_{q^{5}}[2]_{q^{7}}}{[2]_{q}}+t^{2} q^{7}\right)$ \\
\hline$\nu_{3}$ & {$[2]_{q^{4}}[5]_{q} \prod_{i=1}^{2}\left(1+t q^{e_{i}}\right) \prod_{i=1}^{2}\left(q^{e_{i}}+t\right)$.} \\
& $\left.+t[2]_{q^{2}}\left(1-q-q^{2}+2 q^{3}-q^{5}+q^{6}+q^{10}-q^{11}+2 q^{13}-q^{14}-q^{15}+q^{16}\right)\right)$ \\
\hline
\end{tabular}

\begin{tabular}{|c|c|}
\hline$E_{7}$ & exponents $(1,5,7,9,11,13,17)$ \\
\hline \hline$\nu_{2}$ & $(q+t)[3]_{q^{6}}[7]_{q^{2}} \prod_{i=1}^{4}\left(1+t q^{e_{i}}\right) \cdot$ \\
& $\left(q^{5}+t \frac{[2]_{q^{8}}[10]_{q^{2}}}{[2]_{q^{2}}}+t^{2} q^{11}\right)$ \\
\hline$\nu_{3}$ & {$[5]_{q^{2}}[7]_{q^{2}} \prod_{i=1}^{3}\left(1+t q^{e_{i}}\right) \prod_{i=1}^{2}\left(q^{e_{i}}+t\right) \cdot$} \\
& $\left(\left(q^{7}+q^{9} t^{2}\right) \frac{[10]_{q^{2}}}{[2]_{q^{2}}}+t[2]_{q^{4}}\left(1-2 q^{2}+q^{4}+q^{6}-q^{10}+q^{14}+q^{16}-2 q^{18}+q^{20}\right)\right)$ \\
\hline
\end{tabular}

\begin{tabular}{|c|c|}
\hline$E_{8}$ & exponents $(1,7,11,13,17,19,23,29)$ \\
\hline \hline$\nu_{2}$ & $(q+t)\left(1+q^{12}\right)\left(-q^{14}+\sum_{i=1}^{8} q^{e_{i}-1}\right) \prod_{i=1}^{5}\left(1+t q^{e_{i}}\right) \cdot$ \\
& $\left(\left(1+q^{12} t^{2}\right)\left(\left(q^{7}+q^{11}\right)+t\left(1+q^{14}\right)\left(1+q^{16}\right)\right)\right.$ \\
\hline$\nu_{3}$ & {$[7]_{q^{2}}\left(\sum_{i=1}^{8} q^{e_{i}-1}\right) \prod_{i=1}^{4}\left(1+t q^{e_{i}}\right) \prod_{i=1}^{2}\left(q^{e_{i}}+t\right) \cdot$} \\
& $\left(\left(1+q^{6} t^{2}\right)\left(q^{11}-q^{15}+q^{17}-q^{19}+q^{23}\right)+t\left(1-q^{2}+q^{12}+q^{28}-q^{38}+q^{40}\right)\right)$ \\
\hline$\nu_{4}$ & {$[7]_{q^{2}} \prod_{i=1}^{2}\left(1+t q^{e_{i}}\right) \prod_{i=1}^{2}\left(q^{e_{i}}+t\right) \cdot$} \\
& $\left(\left(1+t^{4}\right) \cdot q^{24}\left(1-q^{2}+q^{4}+q^{6}-q^{8}+q^{10}+q^{12}-2 q^{14}+4 q^{16}-2 q^{20}\right.\right.$ \\
& $\left.+4 q^{22}-2 q^{24}+4 q^{28}-2 q^{30}+q^{32}+q^{34}-q^{36}+q^{38}+q^{40}-q^{42}+q^{44}\right)$ \\
& $+\left(t+t^{3}\right) \cdot q^{11}[2]_{q^{6}}\left(1-q^{4}+q^{6}+3 q^{12}-2 q^{14}+q^{16}+q^{18}+q^{22}+3 q^{24}-q^{26}+q^{28}+2 q^{30}\right.$ \\
& $\left.+2 q^{34}+q^{36}-q^{38}+3 q^{40}+q^{42}+q^{46}+q^{48}-2 q^{50}+3 q^{52}+q^{58}-q^{60}+q^{64}\right)$ \\
& $+t^{2} \cdot\left(1-q^{2}+q^{6}-q^{8}+q^{10}+q^{12}-q^{14}+q^{16}+q^{18}-q^{20}+3 q^{22}+2 q^{24}\right.$ \\
& $-2 q^{26}+5 q^{28}+q^{30}+5 q^{34}+2 q^{36}-q^{38}+9 q^{40}-q^{44}+10 q^{46}-q^{48}$ \\
& $+9 q^{52}-q^{54}+2 q^{56}+5 q^{58}+q^{62}+5 q^{64}-2 q^{66}+2 q^{68}+3 q^{70}-q^{72}$ \\
& $\left.\left.+q^{74}+q^{76}-q^{78}+q^{80}+q^{82}-q^{84}+q^{86}-q^{90}+q^{92}\right)\right)$ \\
\hline
\end{tabular}


Duality, but non-real and non-coincidental reflection groups of rank at least 3.

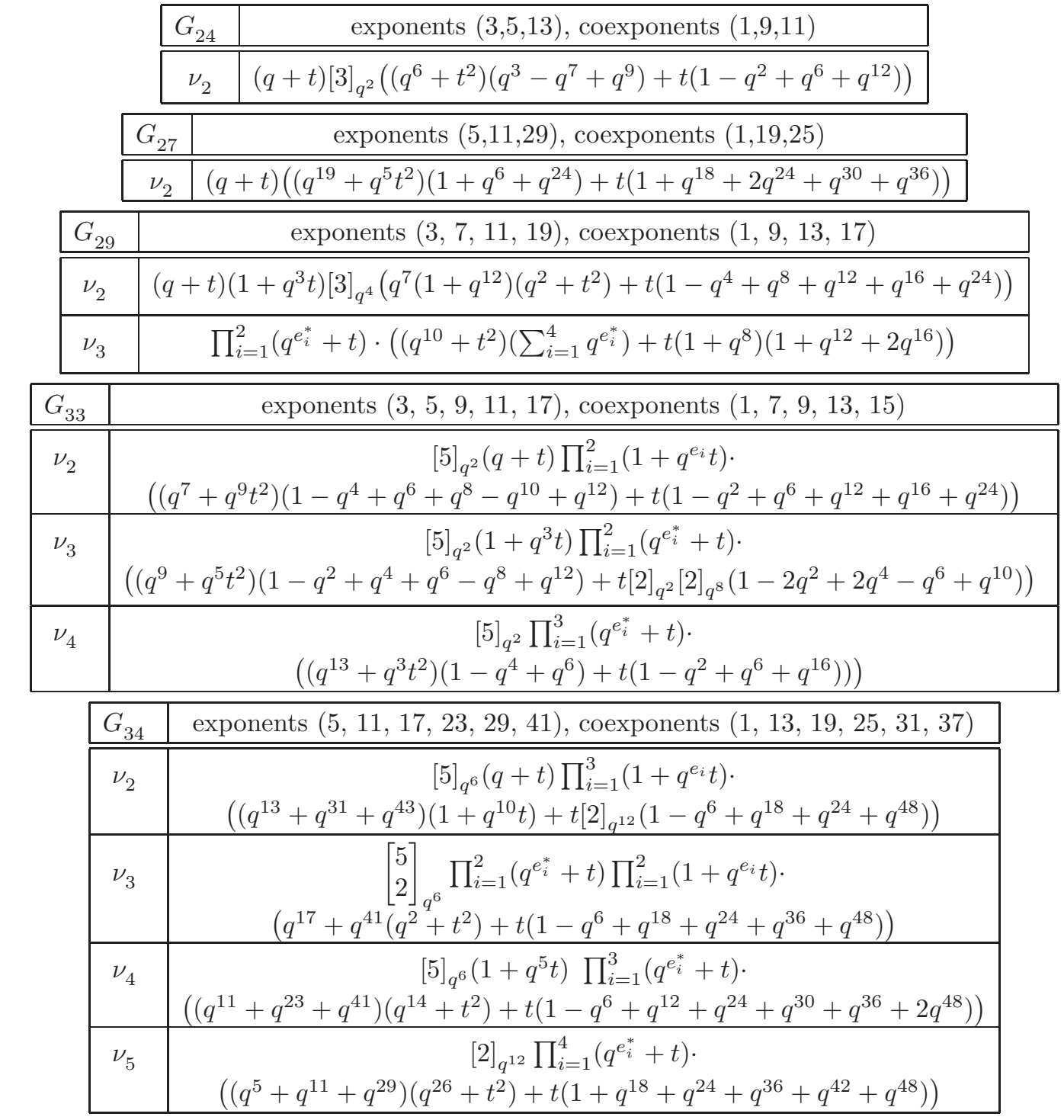

The unique non-duality exceptional reflection group of rank at least 3 .

\begin{tabular}{|c|c|}
\hline$G_{31}$ & exponents $(7,11,19,23)$, coexponents $(1,13,17,29)$ \\
\hline \hline$\nu_{1}$ & {$[2]_{q^{12}}(q+t) \prod_{i=1}^{2}\left(1+q^{e_{i}}\right)\left(1+q^{16}+t\left(q^{19}+q^{23}\right)\right)$} \\
\hline$\nu_{2}$ & $(q+t)\left(1+q^{7} t\right)\left(q^{13}+q^{17}+2 q^{29}+q^{41}+q^{45}\right.$ \\
& $+t\left(1+q^{12}+q^{16}+2 q^{24}+2 q^{28}+2 q^{32}+q^{36}+2 q^{40}\right)$ \\
& $\left.+t^{2}\left(q^{11}+q^{19}+2 q^{23}+q^{27}+q^{35}\right)\right)$ \\
\hline$\nu_{3}$ & {$[2]_{q^{12}} \prod_{i=1}^{3}\left(q^{e_{i}^{*}}+t\right)\left(1+q^{16}+t\left(q^{7}+q^{11}\right)\right)$} \\
\hline
\end{tabular}

\section{REFERENCES}

[1] D. Armstrong, Generalized noncrossing partitions and combinatorics of Coxeter groups. Mem. Amer. Math. Soc. 202 (2009), no. 949.

[2] D. Armstrong, V. Reiner and B. Rhoades, Parking spaces. Adv. Math. 269 (2015), 647-706.

[3] D. Bessis and V. Reiner, Cyclic sieving of noncrossing partitions for complex reflection groups. Ann. Comb. 15 (2011), 197-222.

[4] B. Briggs, Matrix Factorisations Arising From Well-Generated Complex Reflection Groups, arXiv:1704.05966 
[5] M. Broué, Introduction to complex reflection groups and their braid groups. Lecture Notes in Mathematics 1988. SpringerVerlag, Berlin, 2010.

[6] C. Chevalley, Invariants of finite groups generated by reflections. Amer. J. Math. 77 (1955), 778-782.

[7] S. Fomin and N. Reading, Generalized cluster complexes and Coxeter combinatorics. Int. Math. Res. Not. (2005), no. 44, 2709-2757.

[8] S. Fomin and A. Zelevinsky, Y-systems and generalized associahedra. Ann. of Math. (2) 158 (2003), 977-1018.

[9] G. Gasper and M. Rahman, Basic hypergeometric series, Second edition. Encyclopedia of Mathematics and its Applications 96. Cambridge University Press, Cambridge, 2004.

[10] I.G. Gordon, and S. Griffeth, Catalan numbers for complex reflection groups. Amer. J. Math. 134 (2012), 1491-1502.

[11] D. Grinberg and V. Reiner, Hopf algebras in combinatorics, arXiv:1409.8356

[12] M.D. Haiman, Conjectures on the quotient ring by diagonal invariants. J. Algebraic Combin. 3 (1994), 17-76.

[13] Z. Hamaker, R. Patrias, O. Pechenik, N. Williams, Doppelgängers: bijections of plane partitions, $\operatorname{arXiv:1602.05535}$

[14] A. Gyoja, K. Nishiyama, H. Shimura, Invariants for representations of Weyl groups and two-sided cells. J. Math. Soc. Japan 51 (1999), 1-34.

[15] M. Hochster and J.A. Eagon, Cohen-Macaulay rings, invariant theory, and the generic perfection of determinantal loci. Amer. J. Math. 93 (1971), 10201058.

[16] C. Hohlweg, C. Lange, H. Thomas, Permutahedra and generalized associahedra. Adv. Math. 226 (2011), 608-640.

[17] Y. Ito and S. Okada, On the existence of generalized parking spaces for complex reflection groups, arxiv.1508.06846.

[18] R. Kane, Reflection groups and invariant theory, CMS Books in Mathematics 5, Springer-Verlag, New York, 2001.

[19] A.A. Kirillov and I.M. Pak, Covariants of the symmetric group and its analogues in A. Weil algebras. Funktsional. Anal. $i$ Prilozhen. 24 (1990), 9-13; translation in Funct. Anal. Appl. 24 (1990), 172-176 (1991).

[20] K. Koike, Poincaré series on symmetric and alternating tensors for irreducible representations of imprimitive complex reflection groups. J. Algebra 169 (1994), 541-551.

[21] I.G. Macdonald, Symmetric functions and Hall polynomials, 2nd edition. Oxford Classic Texts in the Physical Sciences. The Clarendon Press, Oxford University Press, New York, 2015.

[22] A.R. Miller, Foulkes characters for complex reflection groups, Proc. AMS 143 (2015) 3281-3293.

[23] Walls in Milnor fiber complexes, arXiv:1710.03069

[24] V.F. Molchanov, Poincaré series of representations of finite groups that are generated by reflections. Funktsional. Anal. $i$ Prilozhen. 26 (1992), no. 2, 82-85; translation in Funct. Anal. Appl. 26 (1992), no. 2, 143-145.

[25] P. Orlik and L. Solomon, Unitary reflection groups and cohomology. Invent. Math. 59 (1980), 77-94.

[26] P. Orlik and H. Terao, Arrangements of hyperplanes. Grundlehren der Mathematischen Wissenschaften 300. SpringerVerlag, Berlin, 1992.

[27] N. Reading, Cambrian lattices. Adv. Math. 205 (2006), no. 2, 313-353.

[28] V. Reiner and A.V. Shepler, Invariant derivations and differential forms for reflection groups, to appear in Proc. Lond. Math. Soc.; available at arXiv:1612.01031

[29] V. Reiner and E. Sommers, Weyl group q-Kreweras numbers and cyclic sieving, Ann. Comb. 22 (2018), 819-874.

[30] K. Saito, T. Yano, J. Sekiguchi, On a certain generator system of the ring of invariants of a finite reflection group. Comm. Algebra 8 (1980), 373-408.

[31] G.C. Shephard and J.A. Todd, Finite unitary reflection groups. Canadian J. Math. 6, (1954), 274-304.

[32] A. Shepler, Semi-invariants of finite reflection groups. J. Algebra 220 (1999), no. 1, 314-326.

[33] A. Shepler, Generalized exponents and forms. J. Algebraic Combin., 22 (2005), no. 1, 115-132.

[34] A. Shepler and H. Terao, Logarithmic forms and anti-invariant forms of reflection groups, In Arrangements Tokyo 1998, M. Falk and H. Terao, eds. (Tokyo: Mathematical Society of Japan, 2000), Adv. Stud. Pure Math., 273-278.

[35] L. Solomon, Invariants of finite reflection groups. Nagoya Math. J. 22 (1963), 57-64.

[36] E. Sommers, Exterior powers of the reflection representation in Springer theory. Transform. Groups 16 (2011), 889-911.

[37] R.P. Stanley, Invariants of finite groups and their applications to combinatorics, Bull. AMS 1 (1979), No. 3, 475-511.

[38] R.P. Stanley, Relative invariants of finite groups generated by pseudoreflections. J. Algebra 49 (1977), 134-148.

[39] J.-Y. Thibon, The inner plethysm of symmetric functions and some of its applications. Bayreuth. Math. Schr. 40 (1992), $177-201$. 